\title{
ARTICLE \\ Zero-truncated Compound Poisson integer-valued GARCH models for time series
}

\author{
Esmeralda Gonçalves $^{\mathrm{a} *}$ and Nazaré Mendes-Lopes ${ }^{\mathrm{a}}$ \\ ${ }^{a}$ CMUC, Department of Mathematics, University of Coimbra, Apartado 3008, EC Santa Cruz, \\ 3001-501 Coimbra, Portugal.
}

(v5.0 released February 2015)

\begin{abstract}
Starting from the Compound Poisson INGARCH models, we introduce in this paper a new family of integer-valued models suitable to describe count data without zeros that we name Zero Truncated CP-INGARCH processes. For such class of models, a probabilistic study concerning moments existence, stationarity and ergodicity is developed. The conditional quasimaximum likelihood method is introduced to consistently estimate the parameters of a wide zero truncated compound Poisson subclass of models. The conditional maximum likelihood method is also used to estimate the parameters of ZTCP-INGARCH processes associated to well specified conditional laws. A simulation study that compares some of those estimators and illustrates their finite distance behavior as well as a real-data application conclude the paper.
\end{abstract}

Keywords: Compound Poisson distribution; zero-truncated count time series

AMS Subject Classification: $62 \mathrm{M} 10$

\section{Introduction}

The usual probability distributions describing the integer-valued models present in literature assume, in general, that the count data to be modeled have zero counts, that is, zero is a possible value of their supports. It may however happen that the expected number of zeros according to the probability distribution of the fitted model is not compatible with those actually occurring. We have in this case an inflation situation, or deflation, of the zero value and in order to correct this phenomenon we have to provide for the possibility to mix such distribution with a point probability. This is for example the case of integer-valued zero inflated models, studied in particular in [5], [8] and [10].

There is yet another type of counting series that structurally exclude the zero value. The number of days of hospitalization in an hospital or the number of days of travel of tourists from a certain country in a period of the year are clear examples of count series without zeros. An interesting and dynamical series of count data without zeros is, for instance, the daily number of occupied beds in a central hospital inpatient service. When the structure of the series is such that it makes no sense the occurrence of zeros, the underlying distribution should not include zero in its support. As referred in [7] (p. 174) "some statisticians have encouraged the use of shifted (...) models ... that is, the counts are shifted left so that 1 counts become 0 's, 2's become 1's and so forth.

\footnotetext{
${ }^{*}$ Corresponding author. Email: esmerald@mat.uc.pt
} 
The problem is that the resultant model is not based on data as they in fact exist". The study here developed illustrates clearly that this is not the good approach. So, as suggested by [7], a more adequate possibility to describe such a situation is the truncation of zero in integer-valued models generally compatible with the situation in study. Within count time series analysis, this problem had been already addressed, for instance in [2] considering ZT-Poisson INAR(1) processes.

A class of integer-valued models recently introduced in the literature is the class of integer-valued GARCH processes with compound Poisson conditional distribution (CPINGARCH). This is a very general class of models that is able to respond to situations compatible with all compound Poisson laws among which stand out the Poisson, Negative Binomial, generalized Poisson and Neyman type A laws. In view of the wideness of the family of distributions associated with these processes it is natural to expect that such distributions truncated in zero may be compatible with count models without null results. This fact led us to introduce a new class of models based on the CP-INGARCH class but without the possibility of zeros. We call these new models CP-INGARCH truncated at zero and denote them briefly as ZTCP-INGARCH.

In Section 2 we recall the definition of the compound Poisson model with values in $\mathbb{N}_{0}$ with generalized autoregressive conditional heteroskedasticity and introduce the integervalued compound Poisson truncated at zero model definition (ZTCP-INGARCH). We analyze aspects of its probabilistic structure, namely the existence of moments, and the strict stationarity and ergodicity in a general sub-class. We study then the geometric ZTCP-INGARCH model; this model does not belong to that sub-class but, as we shall see, we are able to establish its second order stationarity. Section 3 includes the consistent estimation of the parameters of the ZTCP-INGARCH model by Poisson conditional quasi-maximum likelihood method as well as the conditional maximum likelihood methodology for particular ZTCP-INGARCH processes, namely those associated to Poisson or to the geometric conditional laws. A simulation study that illustrates and evaluates the estimation methodologies developed concludes this Section. A real-data application, presented in Section 4, and some final conclusions end the paper.

\section{Zero Truncated CP-INGARCH processes}

\subsection{Zero truncated compound Poisson law}

Let us recall that an integer-valued random variable $X$ follows a compound Poisson law with parameter $\lambda, \lambda>0$, if its characteristic function is such that

$$
\Phi_{X}(u)=\exp \left(\lambda\left(\Phi_{Y}(u)-1\right)\right), u \in \mathbb{R},
$$

with $\Phi_{Y}$ the characteristic function of a random variable $Y$, called compounding variable. The corresponding generating function (of probabilities), $g_{X}(u)=E\left(u^{X}\right),|u| \leq 1$, satisfies

$$
g_{X}(u)=\exp \left(\lambda\left(g_{Y}(u)-1\right)\right)
$$

with $g_{Y}$ the generating function of $Y$, and we have $P(X=0)=g_{X}(0)=\exp \left(\lambda\left(g_{Y}(0)-1\right)\right)$ where $g_{Y}(0)=P(Y=0)$.

We say that the nonnegative integer-valued random variable $Z$ follows a compound Poisson law truncated at zero associated to $X$ if its characteristic function is 


$$
\begin{aligned}
\Phi_{Z}(u) & =E\left(e^{i u Z}\right), u \in \mathbb{R} \\
& =E\left(e^{i u X} \mid X \neq 0\right) \\
& =\frac{\sum_{k=1}^{+\infty} e^{i u k} P(X=k)}{P(X \neq 0)} \\
& =\frac{\exp \left(\lambda\left(\Phi_{Y}(u)-1\right)\right)-\exp \left(\lambda\left(g_{Y}(0)-1\right)\right)}{1-\exp \left(\lambda\left(g_{Y}(0)-1\right)\right)} .
\end{aligned}
$$

\subsection{Zero truncated CP-INGARCH model}

Let $X=\left(X_{t}, t \in \mathbb{Z}\right)$ be a nonnegative integer-valued stochastic process and, for $t \in \mathbb{Z}$, let $\underline{X}_{t}$ denote the $\sigma$ - field generated by $\left(X_{t-j}, j \geq 0\right)$.

Definition 2.1 ([4]) The process $X$ is said to follow a compound Poisson GARCH model with values in $\mathbb{N}_{0}$ with orders $p$ and $q(p, q \in \mathbb{N})$ if, for all $t \in \mathbb{Z}$, the characteristic function of $X_{t}$ conditioned on $\underline{X}_{t-1}$ is given by

$$
\Phi_{X_{t} \mid \underline{X}_{t-1}}(u)=\exp \left\{i \frac{\lambda_{t}}{\varphi_{t}^{\prime}(0)}\left[\varphi_{t}(u)-1\right]\right\}, u \in \mathbb{R}
$$

with

$$
E\left(X_{t} \mid \underline{X}_{t-1}\right)=\lambda_{t}=\alpha_{0}+\sum_{j=1}^{p} \alpha_{j} X_{t-j}+\sum_{k=1}^{q} \beta_{k} \lambda_{t-k}
$$

for constants $\alpha_{0}>0, \alpha_{j} \geq 0(j=1, \ldots, p), \beta_{k} \geq 0(k=1, \ldots, q)$ and where $\left(\varphi_{t}, t \in \mathbb{Z}\right)$ is a family of characteristic functions on $\mathbb{R}, \underline{X}_{t-1}-$ measurables, associated to a family of discrete laws with support in $\mathbb{N}_{0}$ and finite mean. $i$ represents the imaginary unit.

In a briefly way, we say that $X$ follows a $C P-I N G A R C H(p, q)$ model.

If $q=1$ and $\beta_{1}=0$, the $C P-I N G A R C H(p, q)$ model is denoted $C P-I N A R C H(p)$.

We note that as $\varphi_{t}$ is the characteristic function of a discrete distribution with support in $\mathbb{N}_{0}$ and finite mean, the derivative of $\varphi_{t}(u)$ at $u=0, \varphi_{t}^{\prime}(0)$, exists and is nonzero.

As the conditional distribution of $X_{t}$ on $\underline{X}_{t-1}$ is a discrete compound Poisson law with support in $\mathbb{N}_{0}$ then for all $t \in \mathbb{Z}$ and conditioned on $\underline{X}_{t-1}, X_{t}$ can be identified in distribution with the random sum

$$
X_{t} \stackrel{d}{=} X_{t, 1}+\ldots+X_{t, N_{t}}
$$

where $N_{t}$ is a random variable following a Poisson distribution with parameter $\frac{\lambda_{t}}{E\left(X_{t, j}\right)}$ and $X_{t, 1}, \ldots, X_{t, N_{t}}$ are discrete and independent random variables, with support contained in $\mathbb{N}_{0}$, independent of $N_{t}$ and having common characteristic function $\varphi_{t}$, with finite mean. The distribution of $X_{t, j}$ is called compounding distribution and we assume $X_{t}$ equal to zero if $N_{t}=0$. This property may be used to generate time series following a CPINGARCH model. 
We can now introduce the definition of the nonzero integer-valued (or zero truncated) generalized autoregressive conditional heteroscedastic compound Poisson model, briefly ZTCP - INGARCH $(p, q)$.

Definition 2.2 The stochastic process $Z=\left(Z_{t}, t \in \mathbb{Z}\right)$ follows a ZTCP $\operatorname{INGARCH}(p, q)$ model if, for any $t \in \mathbb{Z}$, the characteristic function of $Z_{t}$ conditioned on $\underline{Z}_{t-1}$ is given by

$$
\Phi_{Z_{t} \mid \underline{Z}_{t-1}}(u)=\frac{\exp \left\{i \frac{\lambda_{t}}{\varphi_{t}^{\prime}(0)}\left[\varphi_{t}(u)-1\right]\right\}-\exp \left\{i \frac{\lambda_{t}}{\varphi_{t}^{\prime}(0)}\left[g_{t}(0)-1\right]\right\}}{1-\exp \left\{i \frac{\lambda_{t}}{\varphi_{t}^{\prime}(0)}\left[g_{t}(0)-1\right]\right\}}, u \in \mathbb{R}
$$

with

$$
\lambda_{t}=\alpha_{0}+\sum_{j=1}^{p} \alpha_{j} Z_{t-j}+\sum_{k=1}^{q} \beta_{k} \lambda_{t-k}
$$

where $\alpha_{0}>0, \alpha_{j} \geq 0(j=1, \ldots, p), \beta_{k} \geq 0(k=1, \ldots, q)$ and $\left(\varphi_{t}, t \in \mathbb{Z}\right)$ is a family of characteristic functions on $\mathbb{R}, \underline{Z}_{t-1}-$ measurable, associated to a family of discrete laws with support in $\mathbb{N}_{0}$ and finite mean and where $\left(g_{t}, t \in \mathbb{Z}\right)$ is the corresponding family of probability generating functions.

If $q=1$ and $\beta_{1}=0$, the $Z T C P-\operatorname{INGARCH}(p, q)$ model is denoted ZTCP $\operatorname{INARCH}(p)$.

In order to assure that $\lambda_{t}$ is $\underline{Z}_{t-1}-$ measurable we consider, in what follows, $\sum_{k=1}^{q} \beta_{k}<1$.

Example 2.3 a) Considering $\varphi_{t}$ the characteristic function of the Dirac law in $\{1\}$, that is, $\varphi_{t}(u)=\exp (i u), u \in \mathbb{R}$, whose probability generating function is $g_{t}(u)=u, u \in \mathbb{R}$, we obtain for $Z$ the ZT Poisson INGARCH model.

b) Similarly, considering any of the Compound Poisson INGARCH models highlighted in [4], such as the generalized Poisson, the negative binomial (unless a scale factor), DINARCH, GEOMP, GEOMP2 and NTA models, among others, we get in this way the corresponding Zero truncated Compound Poisson INGARCH model.

In the following Figures 1 and 2 we present the trajectories and the basic descriptives of a series $X$ following a $C P-I N G A R C H(1,1)$ model with Poisson conditional law with $\lambda_{t}=0.2+0.5 X_{t-1}+0.3 \lambda_{t-1}$, and of a $Z$ process following the $\operatorname{ZTCP}-\operatorname{INGARCH}(1,1)$ model with the same parameter values in the corresponding $\lambda_{t}$, which illustrate the probabilistic changes related with the zero truncation. In fact, the empirical distributions of the shifted process $X+1$ and of $Z$ are clearly different, even in mean. Obviously, this difference will be more evident with the increasing of the probability of zero occurrences. These series were generated using the algorithm included in Appendix C.

From the relations between the characteristic function and the moments of the corresponding probability law we deduce

$$
E\left(Z_{t} \mid \underline{Z}_{t-1}\right)=\frac{\lambda_{t}}{1-\exp \left\{i \frac{\lambda_{t}}{\varphi_{t}^{\prime}(0)}\left[g_{t}(0)-1\right]\right\}}
$$



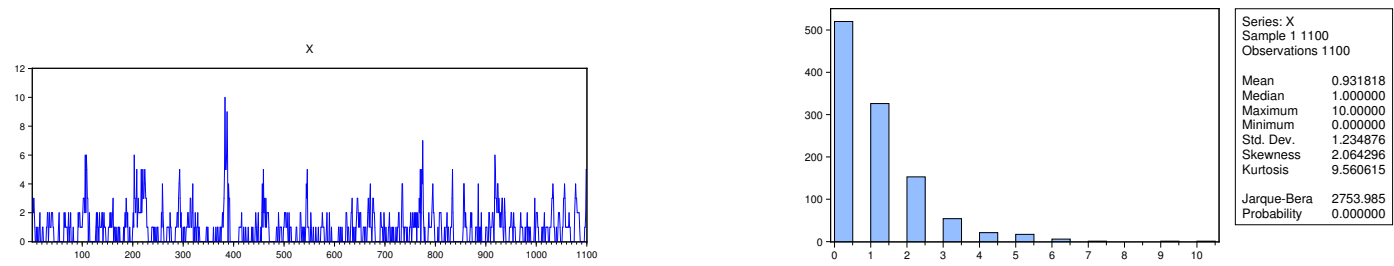

Figure 1. $X$ series following a $C P-I N G A R C H$ model: time plot and principal descriptive summaries
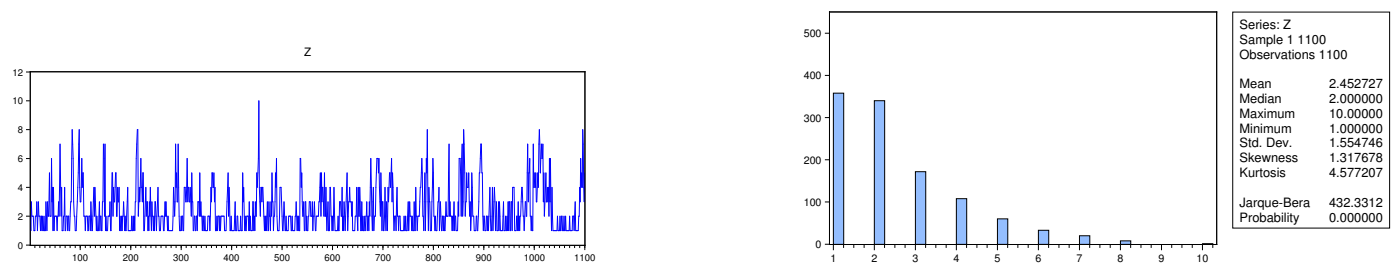

Figure 2. $Z$ series following a $Z T C P-I N G A R C H$ model: time plot and principal descriptive summaries

$$
E\left(Z_{t}^{2} \mid \underline{Z}_{t-1}\right)=\frac{\lambda_{t}^{2}-i \lambda_{t} \frac{\varphi_{t}^{\prime \prime}(0)}{\varphi_{t}^{\prime}(0)}}{1-\exp \left\{i \frac{\lambda_{t}}{\varphi_{t}^{\prime}(0)}\left[g_{t}(0)-1\right]\right\}} .
$$

We note that $E\left(Z_{t}^{k} \mid \underline{Z}_{t-1}\right)$ is a function of $\lambda_{t}^{k}, \lambda_{t}^{k-1}, \ldots, k \in \mathbb{N}$.

\subsection{Probabilistic structure}

The general probabilistic study presented in the subsections 2.3.1 and 2.3.2 is developed within the subclass of $Z T C P-I N G A R C H$ models for which $\varphi_{t}, t \in \mathbb{Z}$, are deterministic functions. In subsection 2.3.3 a particular $Z T C P-I N G A R C H$ model with random $\varphi_{t}$ functions is considered.

\subsubsection{Moments}

Let us begin by establishing that the moments existence of one of the processes involved on the model definition, $Z$ and $\lambda$, determines the moments existence of the other.

Proposition 2.4 If $Z=\left(Z_{t}, t \in \mathbb{Z}\right)$ is a solution of the model (1) then $E\left(Z_{t}\right)$ exists if and only if $E\left(\lambda_{t}\right)$ exists.

Proof. Let us assume that $E\left(\lambda_{t}\right)$ exists. As $g_{t}(0)$ is a probability value, $\varphi_{t}^{\prime}(1)>0$ and $\lambda_{t} \geq \alpha_{0}$, we deduce

$$
E\left(\frac{\lambda_{t}}{1-\exp \left\{\frac{i \lambda_{t}}{\varphi_{t}^{\prime}(1)}\left[g_{t}(0)-1\right]\right\}}\right) \leq E\left(\frac{\lambda_{t}}{1-\exp \left\{\frac{i \alpha_{0}}{\varphi_{t}^{\prime}(1)}\left[g_{t}(0)-1\right]\right\}}\right)=\frac{1}{1-\exp \left\{\frac{i \alpha_{0}}{\varphi_{t}^{\prime}(1)}\left[g_{t}(0)-1\right]\right\}} E\left(\lambda_{t}\right)
$$

and so

$$
E\left(Z_{t}\right)=E\left[E\left(Z_{t} \mid \underline{Z}_{t-1}\right)\right]=E\left(\frac{\lambda_{t}}{1-\exp \left\{\frac{i \lambda_{t}}{\varphi_{t}^{(1)}}\left[g_{t}(0)-1\right]\right\}}\right)
$$


exists. Otherwise, if $E\left(Z_{t}\right)$ exists it is enough to take into account that

$$
\lambda_{t} \leq \frac{\lambda_{t}}{1-\exp \left\{\frac{i \lambda_{t}}{\varphi_{t}^{\prime}(1)}\left[g_{t}(0)-1\right]\right\}}
$$

to conclude that $E\left(\lambda_{t}\right)$ exists.

Moreover, it is easily proved that

$$
E\left(Z_{t}^{k} \mid \underline{Z}_{t-1}\right)=\frac{R\left(\lambda_{t}^{k}, \lambda_{t}^{k-1}, \ldots\right)}{1-\exp \left\{\frac{i \lambda_{t}}{\varphi_{t}^{\prime}(1)}\left[g_{t}(0)-1\right]\right\}}
$$

with $R$ a linear function of $\lambda_{t}^{k}, \lambda_{t}^{k-1}, \ldots$; so it is clear that if the $k$ order moment, $k \in \mathbb{N}$, of one of the processes, $Z$ or $\lambda$, exists so does the $k$ order moment of the other. We note that the existence of such moments requires a priori the $k$ - order differentiability of the $\varphi_{t}$ function.

\subsubsection{Existence of a strictly stationary and ergodic solution}

In order to study the stationarity of the truncated process let us assume that the determinist functions $\varphi_{t}$ are independent of $t$. So we consider in this subsection $\varphi_{t}=\varphi$ and, accordingly, $g_{t}=g$. In these conditions and if $\sum_{j=1}^{p} \alpha_{j}+\sum_{k=1}^{q} \beta_{k}<1$, the $C P-I N G A R C H$ model has a strictly stationary and ergodic solution ([4], Theorem 5$), X^{*}=\left(X_{t}^{*}, t \in \mathbb{Z}\right)$, with characteristic function of the law of $X_{t}^{*}$ conditioned on $X_{t-1}^{*}$ given by

$$
\Phi_{X_{t}^{*} \mid \underline{X}_{t-1}^{*}}(u)=\exp \left\{i \frac{\lambda_{t}^{*}}{\varphi^{\prime}(0)}[\varphi(u)-1]\right\}
$$

with conditioned expectation $E\left(X_{t}^{*} \mid \underline{X}_{t-1}^{*}\right)=\lambda_{t}^{*}=\alpha_{0}+\sum_{j=1}^{p} \alpha_{j} X_{t-j}^{*}+\sum_{k=1}^{q} \beta_{k} \lambda_{t-k}^{*}$ and we can define the process $Z^{*}=\left(Z_{t}^{*}, t \in \mathbb{Z}\right)$ such that

$$
Z_{t}^{*}=X_{t}^{*} \mid X_{t}^{*}>0 .\left({ }^{1}\right)
$$

This process $Z^{*}=\left(Z_{t}^{*}, t \in \mathbb{Z}\right)$ is a solution of the ZTCP - INGARCH model with $\lambda_{t}=\alpha_{0}+\sum_{j=1}^{p} \alpha_{j} Z_{t-j}^{*}+\sum_{k=1}^{q} \beta_{k} \lambda_{t-k}$ and, as $Z_{t}^{*}$ is a measurable function of a strictly stationary and ergodic process, $Z^{*}$ is also strictly stationary and ergodic. In fact, we note that $Z^{*}=\left(Z_{t}^{*}, t \in \mathbb{Z}\right)$ is a stochastic process whose conditional law is the law of

\footnotetext{
${ }^{1}$ In particular, we know that $E\left(X_{t}^{*}\right)$ exists if and only if $E\left(\lambda_{t}^{*}\right)$ exists and this happens if and only if $\sum_{j=1}^{p} \alpha_{j}+$ $\sum_{k=1}^{q} \beta_{k}<1$. Moreover, $E\left(\lambda_{t}^{*}\right)=E\left(X_{t}^{*}\right)$ is independent of $t$.
} 
$X_{t}^{*} \mid \underline{Z}_{t-1}^{*}$ conditioned on $X_{t}^{*}>0$. So,

$$
\begin{aligned}
E\left(Z_{t}^{*} \mid \underline{Z}_{t-1}^{*}\right) & =E\left[E\left(X_{t}^{*}\left|X_{t}^{*}>0\right| \underline{X}_{t-1}^{*}\right) \mid \underline{Z}_{t-1}^{*}\right] \\
& =E\left[\frac{\lambda_{t}^{*}}{P \underline{X}_{t-1}^{*}\left(X_{t}^{*}>0\right)} \mid \underline{Z}_{t-1}^{*}\right] \\
& =E\left[\frac{\alpha_{0}+\sum_{j=1}^{p} \alpha_{j} X_{t-j}^{*}+\sum_{k=1}^{q} \beta_{k} \lambda_{t-k}^{*}}{1-\exp \left\{\frac{i \lambda_{t}^{*}}{\varphi^{\prime}(0)}[g(0)-1]\right\}} \mid \underline{Z}_{t-1}^{*}\right] \\
& =\frac{\alpha_{0}+\sum_{j=1}^{p} \alpha_{j} Z_{t-j}^{*}+\sum_{k=1}^{q} \beta_{k} \lambda_{t-k}}{1-\exp \left\{\frac{i \lambda_{t}}{\varphi^{\prime}(0)}[g(0)-1]\right\}},
\end{aligned}
$$

that is, $E\left(Z_{t}^{*} \mid \underline{Z}_{t-1}^{*}\right)=\frac{\lambda_{t}}{1-\exp \left\{\frac{i \lambda_{t}}{\varphi^{\prime}(0)}[g(0)-1]\right\}}$.

Furthermore, from the equality $\lambda_{t}=\alpha_{0}+\sum_{j=1}^{p} \alpha_{j} Z_{t-j}^{*}+\sum_{k=1}^{q} \beta_{k} \lambda_{t-k}$ we deduce that

$$
\left(1-\sum_{k=1}^{q} \beta_{k} L^{k}\right) \lambda_{t}=\alpha_{0}+\sum_{j=1}^{p} \alpha_{j} Z_{t-j}^{*}
$$

that is,

$$
\lambda_{t}=\frac{\alpha_{0}}{1-\sum_{k=1}^{q} \beta_{k}}+\sum_{n=0}^{+\infty}\left(\sum_{k=1}^{q} \beta_{k} L^{k}\right)^{n} \sum_{j=1}^{p} \alpha_{j} Z_{t-j}^{*} .
$$

We may now present the following property.

Proposition 2.5 The ZTCP-INGARCH model (1) with $\varphi_{t}=\varphi$ has a strictly stationary and ergodic solution, $Z^{*}$, if $\sum_{j=1}^{p} \alpha_{j}+\sum_{k=1}^{q} \beta_{k}<1$. Moreover, if $Z^{*}$ is a strictly stationary and ergodic solution then $\lambda^{*}$ is also a strictly stationary and ergodic process.

If $Z$ is stationary in mean the same happens to $\lambda$ and we have the following relation between the corresponding means:

$$
\begin{aligned}
\left(1-\sum_{k=1}^{q} \beta_{k}\right) E\left(\lambda_{t}\right) & =\alpha_{0}+\sum_{j=1}^{p} \alpha_{j} E\left(Z_{t}\right) \\
& \Leftrightarrow E\left(\lambda_{t}\right)=\frac{\alpha_{0}}{1-\sum_{k=1}^{q} \beta_{k}}+\frac{\sum_{j=1}^{p} \alpha_{j}}{1-\sum_{k=1}^{q} \beta_{k}} E\left(Z_{t}\right) .
\end{aligned}
$$

Example 2.6 It is clear that every ZT process associated to a strictly stationary and ergodic process is also strictly stationary and ergodic. In particular, the ZT processes associated to the models INGARCH $\left(g_{t}(u)=u\right.$ ), NB-DINARCH (with $\varphi_{t}$ the characteristic function of the logarithmic law with parameter $\left.\frac{\alpha-1}{\alpha}, \alpha>0\right)$, NTA-INGARCH (where $\varphi_{t}$ is the characteristic function of the Poisson law with parameter $\phi, \phi>0$ ) 
and GEOMP2-INGARCH (with $\varphi_{t}$ the characteristic function of the geometric law with parameter $p, p \in] 0,1[)$ such that $\sum_{j=1}^{p} \alpha_{j}+\sum_{k=1}^{q} \beta_{k}<1$ have strictly stationary and ergodic solutions as they correspond to models with deterministic and independent of $t$ characteristic functions $\varphi_{t}([4])$.

\subsubsection{A Zero truncated geometric INGARCH model}

In this subsection we present a zero truncated CP-INGARCH model, not belonging to the general subclass previously considered, for which some probabilistic properties, namely the second order stationarity, may be established. As this model is based on the geometric law we begin by recalling that this law is a compound Poisson one.

\section{a) Presentation}

The geometric law belongs to the class of compound Poisson laws. In fact, given $p \in$ ] $0,1\left[\right.$, let $\left(Y_{j}, j \geq 1\right)$ be a sequence of i.i.d. random variables with logarithmic distribution with parameter $p$, that is, with probability function given by

$$
P\left(Y_{j}=y\right)=-\frac{(1-p)^{y}}{y \ln p}, y=1,2, \ldots
$$

and let $N$ be a random variable following a Poisson distribution with mean $-\ln p$ and independent of $\left(Y_{j}, j \geq 1\right)$. Then the random variable $X=Y_{1}+\ldots+Y_{N}$ follows a geometric law with parameter $p$, that is,

$$
P(X=x)=p(1-p)^{x}, x=0,1, \ldots
$$

In these conditions, the characteristic function of $X$ is

$$
\Phi_{X}(u)=\exp \left\{\ln \left(1-(1-p) e^{i u}\right)-\ln p\right\}=\frac{p}{1-(1-p) e^{i u}}
$$

taking into account that $\lambda=\ln p$ and $\varphi_{Y}(u)=\frac{\ln \left(1-(1-p) e^{i u}\right)}{\ln p}$.

The geometric INGARCH process $\left(X_{t}, t \in \mathbb{Z}\right)$, introduced in Zhu (2011) as a particular case of the negative binomial model, is obtained considering, conditionally on $\underline{X}_{t-1}$,

$$
X_{t}=Y_{t, 1}+\ldots+Y_{t, N_{t}}
$$

where $Y_{t, 1}, Y_{t, 2}, \ldots$ are i.i.d. random variables with logarithmic distribution with parameter $\frac{1}{1+\lambda_{t}}$, independent of the random variable $N_{t}$ which follows the Poisson law with parameter $\ln \left(1+\lambda_{t}\right)$. The characteristic function of the compounding variables $\left(Y_{t, j}\right)$ is given by

$$
\varphi_{t}(u)=\frac{\ln \left(1-\frac{\lambda_{t}}{1+\lambda_{t}} e^{i u}\right)}{\ln \frac{1}{1+\lambda_{t}}} .
$$

This function is $\underline{X}_{t-1^{-}}$measurable and dependent on $t$. Nevertheless, as we will see, it is possible to study the stationarity of the corresponding truncated model. 
We note that the generating function of $Y_{t, j}$ is $g_{t}(u)=\frac{\ln \left(1-\frac{\lambda_{t}}{1+\lambda_{t}} u\right)}{\ln \frac{1}{1+\lambda_{t}}}$ and so $g_{t}(0)=0$; moreover

$$
\varphi_{t}^{\prime}(0)=\frac{i \lambda_{t}}{\ln \left(1+\lambda_{t}\right)} .
$$

For the ZT Geometric INGARCH model we obtain

$$
\Phi_{Z_{t} \mid \underline{Z}_{t-1}}(u)=\frac{e^{i u}}{1+\lambda_{t}-\lambda_{t} e^{i u}} .
$$

\section{b) Stationarity of the ZT Geometric INGARCH model}

Let us consider any solution $Z$ of the ZT geometric INGARCH model. The conditional moments of orders 1 and 2 of the law of $Z_{t}$ conditioned on $\underline{Z}_{t-1}$ are given by

$$
\begin{gathered}
E\left(Z_{t} \mid \underline{Z}_{t-1}\right)=\lambda_{t}+1 \\
E\left(Z_{t}^{2} \mid \underline{Z}_{t-1}\right)=\left(2 \lambda_{t}+1\right)\left(\lambda_{t}+1\right) .
\end{gathered}
$$

Taking into account the first equality it is easy to establish that the truncated geometric model $Z$ is stationary in mean if and only if

$$
\sum_{j=1}^{p} \alpha_{j}+\sum_{k=1}^{q} \beta_{k}<1
$$

Under this condition, the processes $\lambda$ and $Z$ are both stationary in mean and the corresponding (non conditional) means are

$$
\begin{aligned}
& E\left(Z_{t}\right)=\mu_{Z}=\frac{\alpha_{0}+1-\sum_{k=1}^{q} \beta_{k}}{1-\sum_{j=1}^{p} \alpha_{j}-\sum_{k=1}^{q} \beta_{k}} \\
& E\left(\lambda_{t}\right)=\mu_{Z}-1=\frac{\alpha_{0}+\sum_{j=1}^{p} \alpha_{j}}{1-\sum_{j=1}^{p} \alpha_{j}-\sum_{k=1}^{q} \beta_{k}} .
\end{aligned}
$$

Let us now analyze the second order stationarity of $Z$. Without loss of generality, we take $p=q$ (the coefficients in excess are considered zero). We have

$$
\begin{aligned}
E\left(Z_{t-j} \lambda_{t-k}\right) & =E\left[E\left(Z_{t-j} \mid \underline{Z}_{t-j-1}\right) \lambda_{t-k}\right], \quad \text { if } k \geq j \\
& =E\left(\lambda_{t-j} \lambda_{t-k}\right)+E\left(\lambda_{t-k}\right) \\
E\left(Z_{t-j} \lambda_{t-k}\right) & =E\left[Z_{t-j}\left(E\left(Z_{t-k} \mid \underline{Z}_{t-k-1}\right)-1\right)\right] \\
& =E\left(Z_{t-j} Z_{t-k}\right)-E\left(Z_{t-j}\right), \quad \text { if } k<j .
\end{aligned}
$$

By developing $E\left(Z_{t} Z_{t-h}\right)=E\left[E\left(Z_{t} \mid \underline{Z}_{t-1}\right) Z_{t-h}\right]=E\left[\left(\lambda_{t}+1\right) Z_{t-h}\right]$ for $h \geq 1$, $E\left(\lambda_{t} \lambda_{t-h}\right)$ for $h \geq 0$, and using the stationarity in mean of $Z$, we get 


$$
\begin{aligned}
& E\left(Z_{t}^{2}\right)=C+2\left[\sum_{j=1}^{p} \alpha_{j}^{2} E\left(Z_{t-j}^{2}\right)+\sum_{\substack{j, k=1 \\
j \neq k}}^{p} \alpha_{j} \alpha_{k} E\left(Z_{t-j} Z_{t-k}\right)+\right. \\
& \left.\quad+2 \sum_{j=1}^{p} \sum_{k=1}^{p} \alpha_{j} \beta_{k} E\left(Z_{t-j} \lambda_{t-k}\right)+\sum_{k=1}^{p} \beta_{k}^{2} E\left(\lambda_{t-k}^{2}\right)+\sum_{\substack{j, k=1 \\
j \neq k}}^{p} \beta_{j} \beta_{k} E\left(\lambda_{t-j} \lambda_{t-k}\right)\right]
\end{aligned}
$$

where $C=3 \mu_{\lambda}+1+2 \alpha_{0}^{2}+4 \alpha_{0}\left(\mu_{\lambda}-\alpha_{0}\right)$. We note that

$$
\begin{aligned}
E\left(Z_{t}^{2}\right)=E\left(2 \lambda_{t}^{2}+3 \lambda_{t}+1\right) & \Leftrightarrow E\left(Z_{t}^{2}\right)=2 E\left(\lambda_{t}^{2}\right)+3 \mu_{\lambda}+1 \\
& \Leftrightarrow E\left(\lambda_{t}^{2}\right)=\frac{E\left(Z_{t}^{2}\right)-3 \mu_{\lambda}-1}{2} \\
& \Leftrightarrow E\left(\lambda_{t}^{2}\right)=\frac{E\left(Z_{t}^{2}\right)}{2}-\frac{3 \mu_{Z}-2}{2},
\end{aligned}
$$

which implies that $Z$ is a second order process if and only if the same occurs with $\lambda$.

Analogously to what is done in [4], Proposition 1, we obtain

i) $E\left(Z_{t}^{2}\right)=b_{0}+2 \sum_{j=1}^{p}\left(\alpha_{j}^{2}+\frac{\beta_{j}^{2}+2 \alpha_{j} \beta_{j}}{2}\right) E\left(Z_{t-j}^{2}\right)+$

$$
\left.+4 \sum_{j=1}^{p-1} \sum_{k=j+1}^{p} \alpha_{k}\left(\alpha_{j}+\beta_{j}\right) E\left(Z_{t-j} Z_{t-k}\right)+4 \sum_{j=1}^{p-1} \sum_{k=j+1}^{p} \beta_{k}\left(\alpha_{j}+\beta_{j}\right) E\left(\lambda_{t-j} \lambda_{t-k}\right)\right]
$$

ii) $E\left(Z_{t} Z_{t-h}\right)=b_{1, h}+\left(\alpha_{h}+\frac{\beta_{h}}{2}\right) E\left(Z_{t-h}^{2}\right)+\sum_{j=1}^{h-1}\left(\alpha_{j}+\beta_{j}\right) E\left(Z_{t-j} Z_{t-h}\right)+$

$$
+\sum_{j=h+1}^{p} \alpha_{j} E\left(Z_{t-j} Z_{t-h}\right)+\sum_{j=h+1}^{p} \beta_{j} E\left(\lambda_{t-j} \lambda_{t-h}\right), h \geq 1
$$

iii) $E\left(\lambda_{t} \lambda_{t-h}\right)=b_{2, h}+\frac{\alpha_{h}+\beta_{h}}{2} E\left(Z_{t-h}^{2}\right)+\sum_{j=h+1}^{p} \alpha_{j} E\left(Z_{t-j} Z_{t-h}\right)+$

$$
+\sum_{j=1}^{h-1}\left(\alpha_{j}+\beta_{j}\right) E\left(\lambda_{t-k} \lambda_{t-h}\right)+\sum_{j=h+1}^{p} \beta_{j} E\left(\lambda_{t-j} \lambda_{t-h}\right), h \geq 1
$$

with $b_{0}=\left(3+4 \alpha_{0}\right)\left(\mu_{Z}-1\right)+1-2 \alpha_{0}^{2}-\left(\frac{3 \mu_{Z}-2}{2}\right) \sum_{k=1}^{p}\left(\beta_{k}^{2}+2 \alpha_{k} \beta_{k}\right)+$

$$
\begin{gathered}
+2\left(\mu_{Z}-1\right) \sum_{\substack{j, k=1 \\
j \leq k}}^{p} \alpha_{j} \beta_{k}-2 \mu_{Z} \sum_{\substack{j, k=1 \\
j<k}}^{p} \alpha_{k} \beta_{j} \\
b_{1, h}=\mu_{Z}\left(1+\alpha_{0}-\sum_{k=1}^{h-1} \beta_{k}\right)+\left(\mu_{Z}-1\right) \sum_{k=h}^{p} \beta_{k}-\frac{3 \mu_{Z}-2}{2} \beta_{h} \\
b_{2, h}=\left(\mu_{Z}-1\right)\left(\alpha_{0}+\sum_{j=1}^{h} \alpha_{j}\right)-\mu_{Z} \sum_{j=h+1}^{p} \alpha_{j}-\frac{3 \mu_{Z}-2}{2}\left(\alpha_{h}+\beta_{h}\right) .
\end{gathered}
$$

From these calculations, it is easy to see that the vector $W_{t}$, with dimension $p+q-1$, given by

$$
W_{t}=\left[\begin{array}{l}
E\left(Z_{t}^{2}\right) \\
E\left(Z_{t} Z_{t-1}\right) \\
\cdots \\
E\left(Z_{t} Z_{t-(p-1)}\right) \\
E\left(\lambda_{t} \lambda_{t-1}\right) \\
\cdots \\
E\left(\lambda_{t} \lambda_{t-(q-1)}\right)
\end{array}\right]
$$


satisfies an autoregressive equation of order $\max (p, q)$

$$
W_{t}=b+\sum_{k=1}^{\max (p, q)} B_{k} W_{t-k}
$$

with $b=\left[b_{j}\right]_{j=1, \ldots, p+q-1}$ a real $p+q-1$ dimensional vector such that

$$
b_{j}= \begin{cases}b_{0}, & j=1 \\ \left(1+\alpha_{0}-\sum_{k=1}^{j-2} \beta_{k}\right) \mu_{X}+\left(\mu_{X}-1\right) \sum_{k=j-1}^{p} \beta_{k}-\frac{3 \mu_{X}-2}{2} \beta_{j-1}, & j=2, \ldots, p \\ \left(\alpha_{0}+\sum_{k=1}^{j-p} \alpha_{k}\right)\left(\mu_{X}-1\right)-\mu_{X} \sum_{k=j-p+1}^{p} \alpha_{k}-\frac{3 \mu_{X}-2}{2}\left(\alpha_{j-p}+\beta_{j-p}\right), & j=p+1, \ldots, p+q-1\end{cases}
$$

and $B_{k}(k=1, \ldots, \max (p, q))$ real square $p+q-1$ dimensional matrices. The coefficients of these matrices are equal to those of the matrices obtained for the corresponding non truncated model ([4]).

So, we may write the following proposition.

Proposition 2.7 A zero truncated geometric INGARCH process, Z, stationary in mean is second order stationary if and only if

$$
P(L)=I_{p+q-1}-\sum_{k=1}^{\max (p, q)} B_{k} L^{k}
$$

is a polynomial matrix such that $\operatorname{det} P(z)$ has all its roots outside the unit circle, where $I_{p+q-1}$ is the identity matrix of $p+q-1$ order and $B_{k}(k=1, \ldots, \max (p, q))$ are the matrices present in the autoregressive equation (2).

\section{c) Particular cases}

Let us analyze some particular cases of the zero truncated geometric INGARCH model, namely those with small orders.

c.1) If $p=q=1$ and $\alpha_{1}+\beta_{1}<1$, the matrix $B_{1}$ reduce to the scalar $2\left(\alpha_{1}^{2}+\frac{\beta_{1}^{2}+2 \alpha_{1} \beta_{1}}{2}\right)$ and the necessary and sufficient condition of weak stationarity of $Z$ becomes $\alpha_{1}^{2}+\left(\alpha_{1}+\beta_{1}\right)^{2}<1$. In this case we have

$$
E\left(Z_{t}\right)=\mu_{Z}=\frac{\alpha_{0}+1-\beta_{1}}{1-\left(\alpha_{1}+\beta_{1}\right)}
$$

and from $E\left(Z_{t}^{2}\right)=b_{0}+B_{1} E\left(Z_{t-1}^{2}\right)$ we get

$$
E\left(Z_{t}^{2}\right)=\frac{b_{0}}{1-B_{1}}
$$

with $b_{0}=\left(3+4 \alpha_{0}-\alpha_{1} \beta_{1}-\frac{3}{2} \beta_{1}^{2}\right) \mu_{Z}-2\left(\alpha_{0}+1\right)^{2}+\beta_{1}^{2}$ and $B_{1}=\alpha_{1}^{2}+\left(\alpha_{1}+\beta_{1}\right)^{2}$. 
Moreover, for $h \geq 1$,

$$
\begin{aligned}
E\left(Z_{t} Z_{t-h}\right) & =\left(1+\alpha_{0}\right) \mu_{Z}+\alpha_{1} E\left(Z_{t-1} Z_{t-h}\right)+\beta_{1}\left(E\left(\lambda_{t}^{2}\right)+E\left(\lambda_{t-h}\right)\right) \\
& =\left(1+\alpha_{0}\right) \mu_{Z}+\beta_{1}\left(\mu_{Z}-1\right)+\beta_{1}\left(\frac{E\left(Z_{t}^{2}\right)}{2}-\frac{3 \mu_{Z}-2}{2}\right)+\alpha_{1} E\left(Z_{t-1} Z_{t-h}\right)
\end{aligned}
$$

and we deduce

$$
\begin{aligned}
E\left(Z_{t} Z_{t-h}\right) & =\left[\left(1+\alpha_{0}\right) \mu_{Z}+\beta_{1}\left(\mu_{Z}-1\right)+\frac{\beta_{1}}{2} E\left(Z_{t}^{2}\right)-\frac{\beta_{1}}{2}\left(3 \mu_{Z}-2\right)\right]\left(\frac{1-\alpha_{1}^{h}}{1-\alpha_{1}}\right)+\alpha_{1}^{h} E\left(Z_{t}^{2}\right) \\
& =\left[\left(1+\alpha_{0}+\beta_{1}\right) \mu_{Z}-\frac{3 \beta_{1}}{2} \mu_{Z}\right]\left(\frac{1-\alpha_{1}^{h}}{1-\alpha_{1}}\right)+\left[\frac{\beta_{1}}{2}\left(\frac{1-\alpha_{1}^{h}}{1-\alpha_{1}}\right)+\alpha_{1}^{h}\right] E\left(Z_{t}^{2}\right) \\
& =\left(1+\alpha_{0}-\frac{\beta_{1}}{2}\right)\left(\frac{1-\alpha_{1}^{h}}{1-\alpha_{1}}\right) \mu_{Z}+\left[\frac{\beta_{1}+\left(-2 \alpha_{1}-\beta_{1}+2\right) \alpha_{1}^{h}}{2\left(1-\alpha_{1}\right)}\right] E\left(Z_{t}^{2}\right) .
\end{aligned}
$$

The autocovariance function of $Z, \gamma(h)=\operatorname{Cov}\left(Z_{t} Z_{t-h}\right), h \geq 0$, follows from these equalities.

Remark 2.8 We note that the expressions obtained may be used to estimate the parameters of the model by the moments method in a simple but consistent way. If $\beta_{1}=0$, for example, the estimators of $\alpha_{0}$ and $\alpha_{1}$ are

$$
\widehat{\alpha}_{0}=\bar{Z}\left(1-\widehat{\alpha}_{1}\right)-1, \quad \widehat{\alpha}_{1}=\sqrt{\frac{\bar{Z}(1-\bar{Z})+S_{Z}^{2}}{2 S_{Z}^{2}}}
$$

where $\bar{Z}$ and $S_{Z}^{2}$ denote, respectively, the empirical mean and variance of a $n$-sample $\left(Z_{1}, \ldots, Z_{n}\right)$ of the process $Z$.

c.2) If $p=q=2, B_{1}$ and $B_{2}$ are 3 -order matrices equal to

$$
\begin{gathered}
B_{1}=\left[\begin{array}{ccc}
2 \alpha_{1}^{2}+\beta_{1}^{2}+2 \alpha_{1} \beta_{1} & 4 \alpha_{2}\left(\alpha_{1}+\beta_{1}\right) & 4 \beta_{2}\left(\alpha_{1}+\beta_{1}\right) \\
\alpha_{1}+\frac{\beta_{1}}{2} & \alpha_{2} & \beta_{2} \\
\frac{\alpha_{1}+\beta_{1}}{2} & \alpha_{2} & \beta_{2}
\end{array}\right] \\
B_{2}=\left[\begin{array}{cccc}
2 \alpha_{2}^{2}+\beta_{2}^{2}+2 \alpha_{2} \beta_{2} & 0 & 0 \\
0 & 0 & 0 \\
0 & 0 & 0
\end{array}\right]
\end{gathered}
$$

and we get

$$
\begin{aligned}
\operatorname{det} P(z)= & 1-\left(\left(\alpha_{1}+\beta_{1}\right)^{2}+\alpha_{2}+\beta_{2}+\alpha_{1}^{2}\right) z+ \\
& +\left(\left(\alpha_{1}+\beta_{1}\right)^{2}\left(\alpha_{2}+\beta_{2}\right)+\left(\alpha_{2}+\beta_{2}\right)^{2}+\alpha_{2}^{2}-\alpha_{1}^{2} \beta_{2}+\alpha_{1}^{2} \alpha_{2}+2 \alpha_{1} \alpha_{2} \beta_{1}\right) z^{2}+ \\
& +\left(\left(\alpha_{2}+\beta_{2}\right)^{3}+\alpha_{2}^{2}\left(\alpha_{2}+\beta_{2}\right)\right) z^{3} .
\end{aligned}
$$

If, in particular, $\alpha_{1}=\beta_{1}=0$ then the roots of $\operatorname{det} P(z)=0$ are

$$
z_{1}=\frac{1}{\alpha_{2}+\beta_{2}}, z_{2}=\frac{1}{\sqrt{\left(\alpha_{2}+\beta_{2}\right)^{2}+\alpha_{2}^{2}}}, z_{3}=-z_{2},
$$

and the necessary and sufficient condition of weak stationarity of $Z$ becomes $\left(\alpha_{2}+\beta_{2}\right)^{2}+$ $\alpha_{2}^{2}<1$. 


\section{Parameter estimation}

The parameter estimation of a ZTCP-INGARCH process is the goal of this Section. Firstly we consider a general estimation methodology, the Poisson conditional quasimaximum likelihood [1], which is applicable to the wide class of ZTCP-INGARCH models with $\varphi_{t}$ deterministic and independent of $t$. The following two subsections are dedicated to present the conditional maximum likelihood approach in two different situations; namely, we consider firstly that the conditional law is a ZT Poisson one and, after, a ZT geometric. We stress that in this last case we estimate a ZTCP-INGARCH model for which $\varphi_{t}$ is a non-deterministic measurable function of $\lambda_{t}$.

\subsection{Zero truncated CP-INGARCH process: Poisson conditional quasi-maximum likelihood estimation}

Following [1] we may deduce the Poisson quasi-maximum likelihood estimator of the parameter vector of a stochastic process $Z$ following a ZTCP-INGARCH model with $\varphi_{t}$ deterministic and independent of $t$. So, the estimator is deduced considering that the conditional law of $Z_{t}$ given $\underline{Z}_{t-1}$ is a Poisson one.

So, we have $n$ observations, $Z_{1}, \ldots, Z_{n}$, of a strictly and ergodic time series valued in $\mathbb{N}$ following a ZTCP-INGARCH model (1) with deterministic and independent of time $\varphi_{t}=\varphi$ functions, such that

$$
E\left(Z_{t} \mid \underline{Z}_{t-1}\right)=m\left(Z_{t-1}, Z_{t-2}, \ldots ; \theta_{0}, K_{0}\right)=\frac{\lambda_{t}}{1-\exp \left\{i \frac{\lambda_{t}}{\varphi^{\prime}(0)}[g(0)-1]\right\}}=\frac{\lambda_{t}}{1-\exp \left(-K_{0} \lambda_{t}\right)}
$$

with $\theta_{0}=\left(\alpha_{00}, \alpha_{01}, \ldots, a_{0 p}, \beta_{01}, \ldots, \beta_{0 q}\right)$ unknown belonging to some parameter compact subset, $\Theta$, of $] 0,+\infty\left[\times\left[0,+\infty\left[{ }^{p+q}, K_{0}=\frac{i}{\varphi^{\prime}(0)}[1-g(0)]>0\right.\right.\right.$, and where

$$
\lambda_{t}=\lambda_{t}\left(\theta_{0}\right)=\alpha_{00}+\sum_{j=1}^{p} \alpha_{0 j} Z_{t-j}+\sum_{k=1}^{q} \beta_{0 k} \lambda_{t-k}\left(\theta_{0}\right)
$$

with $\sum_{k=1}^{q} \beta_{0 k}<1$

Considering the polynomials $A_{\theta_{0}}(z)=\sum_{j=1}^{p} \alpha_{0 j} z^{j}$ and $B_{\theta_{0}}(z)=1-\sum_{k=1}^{q} \beta_{0 k} z^{k}$, we assume that if $q>0, A_{\theta_{0}}(z)$ and $B_{\theta_{0}}(z)$ have no common roots and that at least one $\alpha_{0 j} \neq 0$ for $j=1, \ldots, p$, and $\beta_{0 q} \neq 0$ if $\alpha_{0 p}=0$.

For all $\left.\varphi=(\theta, K)=\left(\alpha_{0}, \alpha_{1}, \ldots, \alpha_{p}, \beta_{1}, \ldots, \beta_{q}, K\right) \in \Theta \times\right] 0,+\infty[$, the measurable function $m=m\left(Z_{t-1}, Z_{t-2}, \ldots ; \varphi\right)$ is valued in $\left(\alpha_{0},+\infty\right)$, since

$$
m=\frac{\lambda_{t}}{1-\exp \left(-K \lambda_{t}\right)} \geq \lambda_{t} \geq \alpha_{0} .
$$

We also assume the existence of $E\left(Z_{t}\right)^{1+\varepsilon}$, for some $\varepsilon>0$, and that the conditional distribution of $Z_{t}$ is non degenerated.

For all $\varphi \in \Theta \times] 0,+\infty\left[, z_{0} \in \mathbb{N}\right.$ and $t \geq 1$, we consider 


$$
m_{t}(\varphi)=m\left(Z_{t-1}, Z_{t-2}, \ldots ; \varphi\right) \text { and } \widetilde{m}_{t}(\varphi)=m\left(Z_{t-1}, Z_{t-2}, \ldots, Z_{1}, z_{0}, z_{0}, \ldots ; \varphi\right) .
$$

The choice of $z_{0}$ for the unknown initial values $Z_{0}, Z_{-1}, \ldots$ involved in $m_{t}(\varphi)$ is asymptotically unimportant since we have a.s.

$$
\lim _{t \rightarrow+\infty} a_{t}=0 \text { and } \lim _{t \rightarrow+\infty} Z_{t} a_{t}=0, \text { where } a_{t}=\sup _{\varphi \in \Theta \times] 0,+\infty[}\left|\widetilde{m}_{t}(\varphi)-m_{t}(\varphi)\right| .
$$

In fact,

$$
\begin{aligned}
\left|\widetilde{m}_{t}(\varphi)-m_{t}(\varphi)\right| & =\left|\frac{\lambda_{t}}{1-\exp \left(-K \lambda_{t}\right)}-\frac{\tilde{\lambda}_{t}}{1-\exp \left(-K \tilde{\lambda}_{t}\right)}\right| \\
& \leq\left|\frac{\exp \left(-K \lambda_{t}\right)-\exp \left(-K \tilde{\lambda}_{t}\right)}{\left(1-\exp \left(-K \lambda_{t}\right)\right)\left(1-\exp \left(-K \tilde{\lambda}_{t}\right)\right)}\right|\left|\lambda_{t}\right|+\frac{\left|\lambda_{t}-\widetilde{\lambda}_{t}\right|}{M}
\end{aligned}
$$

where $M=1-\exp \left(-K \alpha_{0}\right)$. So,

$$
\left|\widetilde{m}_{t}(\varphi)-m_{t}(\varphi)\right| \leq \frac{\left|\exp \left(-K \lambda_{t}\right)-\exp \left(-K \widetilde{\lambda}_{t}\right)\right|}{M^{2}}\left|\lambda_{t}\right|+\frac{\left|\lambda_{t}-\widetilde{\lambda}_{t}\right|}{M} .
$$

Noting that equation (3) is similar to that satisfied by the volatility in a $\operatorname{GARCH}(p, q)$ model, the arguments used by [3] to show (7.30), p. 157, give

$$
\sup _{\theta \in \Theta}\left|\lambda_{t}(\theta)-\tilde{\lambda}_{t}(\theta)\right| \leq R \rho^{t}
$$

where $R$ and $\rho$ denote generic quantities such that $R>0$ and $\rho \in(0,1)$.

Moreover the inequality $\left|\lambda_{t}(\theta)-\widetilde{\lambda}_{t}(\theta)\right| \leq R \rho^{t}$ is equivalent to

$\exp \left(-K \widetilde{\lambda}_{t}(\theta)\right)\left[\exp \left(-K R \rho^{t}\right)-1\right] \leq \exp \left[-K \lambda_{t}(\theta)\right]-\exp \left[-K \widetilde{\lambda}_{t}(\theta)\right] \leq \exp \left(-K \widetilde{\lambda}_{t}(\theta)\right)\left[\exp \left(K R \rho^{t}\right)-1\right]$

As $\exp \left(-K \widetilde{\lambda}_{t}(\theta)\right) \leq 1$ and $\lim _{t \rightarrow+\infty}\left[\exp \left( \pm K R \rho^{t}\right)-1\right]=0$, we deduce that

$$
\exp \left[-K \lambda_{t}(\theta)\right]-\exp \left[-K \widetilde{\lambda}_{t}(\theta)\right]=o(1), t \rightarrow+\infty .
$$

Thus

$$
\left\{\exp \left[-K \lambda_{t}(\theta)\right]-\exp \left[-K \widetilde{\lambda}_{t}(\theta)\right]\right\} \lambda_{t}(\theta)=o(1)
$$

as, when $t \rightarrow+\infty, \lambda_{t}$ is either a.s. bounded or tends a.s. to $+\infty$, and $\widetilde{\lambda}_{t}$ is asymptotically equivalent to $\lambda_{t}$. Finally we may write

$$
\left|\exp \left[-K \lambda_{t}(\theta)\right]-\exp \left[-K \widetilde{\lambda}_{t}(\theta)\right]\right|\left|\lambda_{t}(\theta)\right|=o(1), t \rightarrow+\infty .
$$

As $\varphi \mapsto m_{t}(\varphi)$ is almost surely continuous and $\left.\Theta \times\right] 0,+\infty[$ is a compact set, a Poisson QML estimator of $\varphi_{0}$ is defined ([6]) as any measurable solution of 


$$
\widehat{\varphi}_{n}=\arg \max _{\varphi \in \Theta \times] 0,+\infty[} \widetilde{L}_{n}(\varphi), \widetilde{L}_{n}(\varphi)=\frac{1}{n} \sum_{t=s+1}^{n} \widetilde{l}_{t}(\varphi)
$$

where $\widetilde{l}_{t}(\varphi)=-\widetilde{m}_{t}(\varphi)+Z_{t} \log \widetilde{m}_{t}(\varphi)$ and $s$ is an integer.

So, the conditions (1)-(6) of [1] are satisfied and we may state the following result establishing the consistency of the Poisson conditional QML estimator of $\varphi$.

Theorem 3.1 Under the previous conditions and if the parameter $\varphi$ is identifiable, the Poisson conditional QML estimator $\widehat{\varphi}_{n}$ defined in (4) satisfies

$$
\lim _{n} \widehat{\varphi}_{n}=\varphi_{0} \text { a.s. }
$$

We observe that when the parameter $K$ is known the identifiability assumption

$$
m_{t}(\theta)=m_{t}\left(\theta_{0}\right) \text { almost surely if and only if } \theta=\theta_{0}
$$

is satisfied, which may be proved using the arguments of [1] (for their relation (10)) and taking also into account that the function $\frac{x}{1-\exp (-K x)}$ is injective, $x>0$. So, in this case the consistency of the Poisson conditional QML estimator of $\theta, \lim _{n} \widehat{\theta}_{n}=\theta_{0}$ a.s., is stated.

In both cases, the study of the asymptotic distribution uses new conditions with nontrivial analysis in this context but, like in [1], the asymptotic normality of this estimator is expected when $\varphi_{0}$ belongs to the interior of $\left.\Theta \times\right] 0,+\infty[$.

\subsection{Zero truncated Poisson INGARCH process: Conditional maximum likelihood estimation}

Using the conditional maximum likelihood methodology we estimate in this subsection the parameter vector of a stochastic process $Z$ following a ZTCP-INGARCH $(p, q)$ model for which $g(u)=u$, that is, the conditional law is a ZT Poisson one.

The generating function of $Z_{t}$ conditioned on $\underline{Z}_{t-1}$ is then

$$
G_{Z_{t} \mid \underline{Z}_{t-1}}(u)=\frac{\exp \left(\lambda_{t}(u-1)\right)-\exp \left(-\lambda_{t}\right)}{1-\exp \left(-\lambda_{t}\right)}
$$

with $\lambda_{t}=\alpha_{0}+\sum_{j=1}^{p} \alpha_{j} Z_{t-j}+\sum_{k=1}^{q} \beta_{k} \lambda_{t-k}$. Thus, the probability function of the conditioned law is

$$
P\left(Z_{t}=k \mid \underline{Z}_{t-1}\right)=\frac{G_{Z_{t} \mid \underline{Z}_{t-1}}^{(k)}(0)}{k !}=\frac{\exp \left(-\lambda_{t}\right)\left(\lambda_{t}\right)^{k}}{\left(1-\exp \left(-\lambda_{t}\right)\right) k !}, k=1,2, \ldots
$$

The conditional likelihood function associated to $n$ observations $Z_{1}, \ldots, Z_{n}$ conditionally to the initial values is

$$
L(\theta)=\prod_{t=1}^{n} \frac{\exp \left(-\lambda_{t}\right)\left(\lambda_{t}\right)^{Z_{t}}}{\left(1-\exp \left(-\lambda_{t}\right)\right) Z_{t} !}
$$

where $\theta=\left(\alpha_{0}, \alpha_{1}, \ldots, \alpha_{p}, \beta_{1}, \ldots, \beta_{q}\right)^{T}=\left(\theta_{0}, \theta_{1}, \ldots, \theta_{p}, \theta_{p+1}, \ldots, \theta_{p+q}\right)^{T}$ 
The log-likelihood function is given by

$$
\begin{aligned}
\mathcal{L}(\theta) & =\log L(\theta)=\sum_{t=1}^{n}\left[Z_{t} \log \left(\lambda_{t}\right)-\lambda_{t}-\log \left(Z_{t} !\right)-\log \left(1-\exp \left(-\lambda_{t}\right)\right)\right] \\
& =\sum_{t=1}^{n} l_{t}(\theta)
\end{aligned}
$$

with $l_{t}(\theta)=Z_{t} \log \left(\lambda_{t}\right)-\lambda_{t}-\log \left(Z_{t} !\right)-\log \left(1-\exp \left(-\lambda_{t}\right)\right)$.

To estimate the true value of $\theta, \theta_{0}$, it is natural to maximize $\mathcal{L}(\theta)$ but, as the estimates has no closed form, numerical optimization methods have to be used, taking into account the initial values to be considered in the computations.

In order to estimate the asymptotic covariance matrix of the conditional maximum likelihood estimator, the first and second derivatives of $l_{t}$ in order to $\theta_{i}, \frac{\partial l_{t}}{\partial \theta_{i}}$ and $\frac{\partial^{2} l_{t}}{\partial \theta_{i} \partial \theta_{j}}$, $0 \leq i, j \leq p+q$ are deduced in Appendix A and the usual information matrix equality

$$
-E\left(\frac{\partial^{2} l_{t}}{\partial \theta_{i} \partial \theta_{j}}\right)=E\left(\frac{\partial l_{t}}{\partial \theta_{i}} \frac{\partial l_{t}}{\partial \theta_{j}}\right)
$$

is stated.

\subsection{Zero truncated Geometric INGARCH process: Conditional maximum likelihood estimation}

Let us consider now the particular model previously referred, associated to a random and dependent of $t$ characteristic function, that is, the stochastic process $Z$ following a Zero Truncated geometric INGARCH model with $\lambda_{t}=\alpha_{0}+\sum_{j=1}^{p} \alpha_{j} Z_{t-j}+\sum_{k=1}^{q} \beta_{k} \lambda_{t-k}$.

Using the conditional maximum likelihood methodology we estimate in this subsection the parameter vector of the stochastic process $Z$.

The probability function of the conditioned law is then

$$
P\left(Z_{t}=k \mid \underline{Z}_{t-1}\right)=\frac{G_{Z_{t} \mid \underline{Z}_{t-1}}^{(k)}(0)}{k !}=\frac{\lambda_{t}^{k-1}}{\left(1+\lambda_{t}\right)^{k}}, k=1,2, \ldots
$$

Thus, the conditional likelihood function associated to $n$ observations $Z_{1}, \ldots, Z_{n}$ conditionally to the initial values is

$$
L(\theta)=\prod_{t=1}^{n} \frac{1}{\lambda_{t}}\left(\frac{\lambda_{t}}{1+\lambda_{t}}\right)^{Z_{t}}
$$

where $\theta=\left(\alpha_{0}, \alpha_{1}, \ldots, \alpha_{p}, \beta_{1}, \ldots, \beta_{q}\right)^{T}=\left(\theta_{0}, \theta_{1}, \ldots, \theta_{p}, \theta_{p+1}, \ldots, \theta_{p+q}\right)^{T}$

The log-likelihood function is given by

$$
\begin{aligned}
\mathcal{L}(\theta) & =\log L(\theta)=\sum_{t=1}^{n}\left[-\log \lambda_{t}+Z_{t} \log \left(\frac{\lambda_{t}}{1+\lambda_{t}}\right)\right] \\
& =\sum_{t=1}^{n} l_{t}(\theta)
\end{aligned}
$$

with $l_{t}(\theta)=-\log \lambda_{t}+Z_{t}\left[\log \lambda_{t}-\log \left(1+\lambda_{t}\right)\right]$. 
The estimates are obtained, as previously, by numerical optimization methods considering convenient initial values. Moreover we deduce in Appendix B the first and second derivatives of $l_{t}$ in order to $\theta_{i}, \frac{\partial l_{t}}{\partial \theta_{i}}$ and $\frac{\partial^{2} l_{t}}{\partial \theta_{i} \partial \theta_{j}}, 0 \leq i, j \leq p+q$ and state the usual information matrix equality

$$
-E\left(\frac{\partial^{2} l_{t}}{\partial \theta_{i} \partial \theta_{j}}\right)=E\left(\frac{\partial l_{t}}{\partial \theta_{i}} \frac{\partial l_{t}}{\partial \theta_{j}}\right)
$$

\subsection{Simulation study}

To implement the estimation methodology and analyze its performance in a concrete situation we consider now a stochastic process $Z$ following a ZT Poisson $\operatorname{INGARCH}(1,1)$ model with $\lambda_{t}=0.8+0.5 Z_{t-1}+0.3 \lambda_{t-1}$. In this case we have $K=1$ as defined in Section 3.1. We generate fifty model replications each one with 1100 observations and, in order to minimize the effect of the initial conditions, we discard for each replication the first 100 observations.

In Table 1 we present the estimates of the model parameters using, respectively, the conditional maximum likelihood and the conditional quasi-maximum likelihood estimation methodologies. We point out that, in the first case, we take the true conditional likelihood that is the corresponding to the ZT Poisson conditional law and, in the second one, we consider a pseudo-conditional likelihood assuming that the conditional distribution is Poisson with parameter $E\left(Z_{t} / Z_{t-1}\right)=\frac{\lambda_{t}}{1-\exp \left(-K \lambda_{t}\right)}$ and $K$ unknown.

We have taken samples of sizes 600 and 1000 for each one of the replications, and we observe, in both cases, an increasing proximity of the estimates and the true values of the model parameter vector as in particular the standard errors reveal (in all tables, four significant decimal digits are displayed).

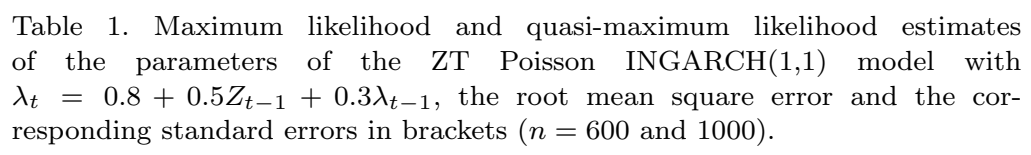

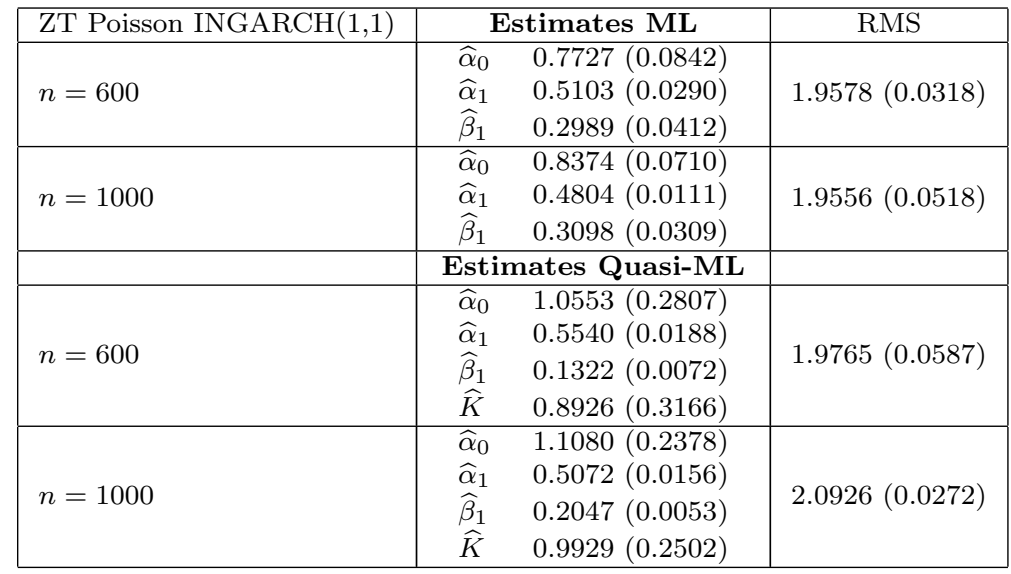

The natural better performance of the conditional maximum likelihood estimator is observed, but the good results also obtained with the conditional quasi-maximum likelihood one reveal a robust estimation alternative with a huge field of application. Its robustness is particularly observed taking into consideration the great proximity, in all 
cases, of the mean square error given by

$$
R M S^{2}=\frac{1}{n} \sum_{t=1}^{n}\left(Z_{t}-\frac{\widehat{\lambda}_{t}}{1-\exp \left(-\widehat{K} \hat{\lambda}_{t}\right)}\right)^{2} .
$$

In order to evaluate the finite distance performance of the conditional likelihood estimator of the parameter vector of a $\mathrm{ZT}$ Geometric INGARCH process we generate now, within the same framework of sample sizes and replications, a stochastic process $Z$ following a ZT Geometric INGARCH(1,1) model with $\lambda_{t}=0.8+0.5 Z_{t-1}+0.3 \lambda_{t-1}$. The estimates obtained for the parameters are presented in the Table 2 . We stress the closeness between the estimates and the true values of the model parameters, when the number of observations increase, particularly assessed by the significant decrease of the corresponding mean square error.

Table 2. Maximum likelihood estimates of the parameters of the ZT Geometric $\operatorname{INGARCH}(1,1)$ model with $\lambda_{t}=0.8+0.5 Z_{t-1}+0.3 \lambda_{t-1}$, the root mean square error and the corresponding standard errors in brackets ( $n=600$ and 1000).

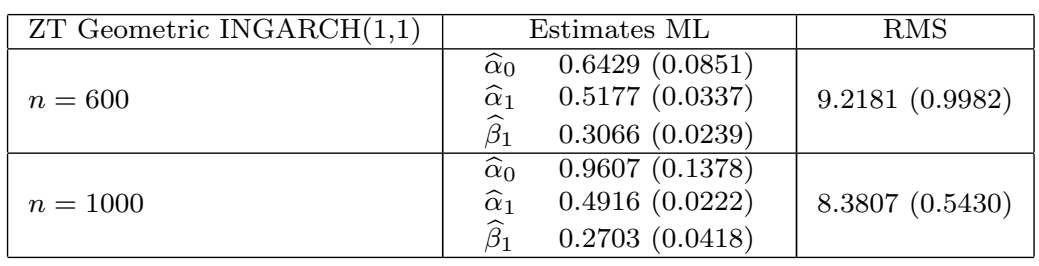

We study also the effect of modeling a sample of 1000 observations generated by the same initial ZT Poisson INGARCH(1,1) process by other models of the ZT Poisson INGARCH class with orders different from the true ones. For the comparison we use the log-likelihood function and Akaike and Schwarz criteria values and we resume this study in Table 3.

Comparing the results with those of the ZT Poisson INGARCH $(1,1)$ model used to generate the observations (line 2 of Table 3 ), we note that in the two ZT Poisson INARCH models considered (lines 3 and 4 of Table 3) the log-likelihood function has smaller values. Moreover the minimum values of the Akaike and Schwarz criteria are as expected obtained for the ZT Poisson INGARCH $(1,1)$. However we note that the ZT Poisson $\operatorname{INARCH}(2)$ model competes well with the true model which is certainly related with the great coefficient of $Z_{t-1}$ in the evolution of $\lambda_{t}$.

We also study the effect of modeling the same observations by the corresponding non truncated INGARCH models and the results, reported in lines 5, 6 and 7 of Table 3, although not strongly different from the previous ones, present the lowest values of the log-likelihood function and the highest ones for Akaike and Schwarz criteria regarding all the models considered.

The small differences between the standard and the zero-truncated Poisson models may be explained by the value of the mean of the count response variable ([8]), greater than 4 , which corresponds to a negligible probability for the occurrence of zero values. In order to see the influence of the mean, we repeat the analysis considering now 1000 observations from a ZT Poisson INGARCH $(1,1)$ model with a smaller mean by considering $\lambda_{t}=$ $0.5+0.3 Z_{t-1}+0.2 \lambda_{t-1}$, whose results are detailed in Table 4 .

We note that in this case the differences between the true model and the non trun- 
Table 3. Conditional maximum likelihood estimates of the parameters of the models, with the corresponding standard errors and probabilities, the log-likelihood function and Akaike and Schwarz criteria.

\begin{tabular}{|c|c|c|c|c|c|c|c|}
\hline Model & \multicolumn{4}{|c|}{ Estimates } & $\log \mathrm{L}$ & Akaike & Schwarz \\
\hline ZT Poisson INGARCH $(1,1)$ & $\begin{array}{l}\widehat{\alpha}_{0} \\
\widehat{\alpha}_{1} \\
\widehat{\beta}_{1}\end{array}$ & $\begin{array}{l}\text { Coeff. } \\
0.7300 \\
0.4938 \\
0.3380\end{array}$ & $\begin{array}{l}\text { Std. Error } \\
0.1974 \\
0.0367 \\
0.0570\end{array}$ & $\begin{array}{l}\text { Prob. } \\
0.0002 \\
0.0000 \\
0.0000\end{array}$ & 2672.787 & -5.3396 & -5.3249 \\
\hline ZT Poisson INARCH(1) & $\begin{array}{l}\widehat{\alpha}_{0} \\
\widehat{\alpha}_{1}\end{array}$ & $\begin{array}{l}\text { Coeff. } \\
1.6435 \\
0.6210\end{array}$ & $\begin{array}{l}\text { Std. Error } \\
0.1315 \\
0.0285\end{array}$ & $\begin{array}{l}\text { Prob. } \\
0.0000 \\
0.0000 \\
\end{array}$ & 2652.876 & -5.3018 & -5.2919 \\
\hline ZT Poisson INARCH(2) & $\begin{array}{l}\widehat{\alpha}_{0} \\
\widehat{\alpha}_{1} \\
\widehat{\alpha}_{2}\end{array}$ & $\begin{array}{l}\text { Coeff. } \\
1.2412 \\
0.5025 \\
0.2057\end{array}$ & $\begin{array}{l}\text { Std. Error } \\
0.1454 \\
0.0360 \\
0.0342\end{array}$ & $\begin{array}{l}\text { Prob. } \\
0.0000 \\
0.0000 \\
0.0000\end{array}$ & 2672.180 & -5.3384 & -5.3236 \\
\hline Poisson INGARCH $(1,1)$ & $\begin{array}{l}\widehat{\alpha}_{0} \\
\widehat{\alpha}_{1} \\
\widehat{\beta}_{1}\end{array}$ & $\begin{array}{l}\text { Coeff. } \\
1.0080 \\
0.4698 \\
0.3201\end{array}$ & $\begin{array}{l}\text { Std. Error } \\
0.2042 \\
0.0375 \\
0.0582\end{array}$ & $\begin{array}{l}\text { Prob. } \\
0.0000 \\
0.0000 \\
0.0000\end{array}$ & 2646.227 & -5.2865 & -5.2717 \\
\hline Poisson INARCH(1) & $\begin{array}{l}\widehat{\alpha}_{0} \\
\widehat{\alpha}_{1}\end{array}$ & $\begin{array}{l}\text { Coeff. } \\
1.8666 \\
0.5910\end{array}$ & $\begin{array}{l}\text { Std. Error } \\
0.1371 \\
0.0291\end{array}$ & $\begin{array}{l}\text { Prob. } \\
0.0000 \\
0.0000\end{array}$ & 2627.707 & -5.2514 & -5.2416 \\
\hline Poisson INARCH(2) & $\begin{array}{l}\widehat{\alpha}_{0} \\
\widehat{\alpha}_{1} \\
\widehat{\alpha}_{2}\end{array}$ & $\begin{array}{l}\text { Coeff. } \\
1.4903 \\
0.4778 \\
0.1953\end{array}$ & $\begin{array}{l}\text { Std. Error } \\
0.1515 \\
0.0368 \\
0.0349\end{array}$ & $\begin{array}{l}\text { Prob. } \\
0.0000 \\
0.0000 \\
0.0000\end{array}$ & 2645.742 & -5.2855 & -5.2708 \\
\hline
\end{tabular}

Table 4. Conditional maximum likelihood estimates of the parameters of the models, with the corresponding standard errors and probabilities, the log-likelihood function and Akaike and Schwarz criteria $\left(\lambda_{t}=0.5+0.3 Z_{t-1}+0.2 \lambda_{t-1}\right)$.

\begin{tabular}{|c|c|c|c|c|c|c|c|}
\hline Model & \multicolumn{4}{|c|}{ Estimates } & Log L & Akaike & Schwarz \\
\hline ZT Poisson INGARCH $(1,1)$ & $\begin{array}{l}\widehat{\alpha}_{0} \\
\widehat{\alpha}_{1} \\
\widehat{\beta}_{1}\end{array}$ & $\begin{array}{l}\text { Coeff. } \\
0.4624 \\
0.2457 \\
0.2858\end{array}$ & $\begin{array}{l}\text { Std. Error } \\
0.1944 \\
0.0503 \\
0.1461\end{array}$ & $\begin{array}{l}\text { Prob. } \\
0.0174 \\
0.0000 \\
0.0504\end{array}$ & -498.9299 & 1.0039 & 1.0186 \\
\hline Poisson INGARCH(1,1) & $\begin{array}{l}\widehat{\alpha}_{0} \\
\widehat{\alpha}_{1} \\
\widehat{\beta}_{1}\end{array}$ & $\begin{array}{l}\text { Coeff. } \\
1.1880 \\
0.1768 \\
0.2070\end{array}$ & $\begin{array}{l}\text { Std. Error } \\
0.2695 \\
0.0662 \\
0.1972\end{array}$ & $\begin{array}{l}\text { Prob. } \\
0.0000 \\
0.0075 \\
0.2937\end{array}$ & -751.0212 & 1.5080 & 1.5228 \\
\hline
\end{tabular}

cated one are significant, namely in which concerns the closeness between the parameters estimates and the true values as the standard errors reinforce. Moreover, taking into account all the criteria used, we verify that in this case the Poisson INGARCH $(1,1)$ model gives clearly worst results than the ZT Poisson $\operatorname{INGARCH}(1,1)$ one. In conclusion, the standard model is not so adequate as the $\mathrm{ZT}$ one introduced in this paper to reproduce the zero truncation characteristic of the initial observations.

\section{Real-data example}

In this section, we want to assess the improvement provided with real data when using a model ZTCP-INGARCH instead of a standard CP-INGARCH. In order to do this let us consider the time series of the quarterly counts of poliomyelitis cases in the United States of America starting from January 1970 and ending in December 1983 (56 observations), obtained in the Forecasting Principles site (http:// www.forecastingprinciples.com). Figure 3 presents the original series and its principal descriptive summaries. In Figure 4 the empirical autocorrelations and partial autocorrelations are displayed. 

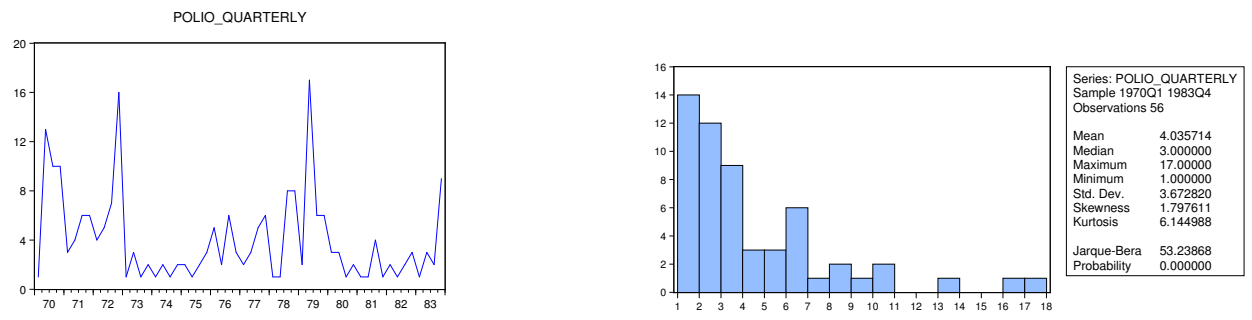

Figure 3. Quarterly poliomyelitis series: time plot and descriptive summaries

We have in fact a strictly positive integer-valued time series whose values are relatively low. The empirical mean and variance of the series are 4.0357 and 13.4896. The empirical analysis of autocorrelation functions seems to show a serial dependence to order 2 of this time series.

\begin{tabular}{|c|c|c|c|c|}
\hline Autocorrelation & Partial Correlation & & $A C$ & PAC \\
\hline ！ り & । 曰 & 1 & 0.213 & 0.213 \\
\hline । & ו صا & 2 & 0.211 & 0.173 \\
\hline 151 & $1\lceil 1$ & 3 & 0.084 & 0.011 \\
\hline 1 口 & 101 & 4 & 0.121 & 0.073 \\
\hline 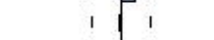 & 101 & 5 & -0.009 & -0.063 \\
\hline 11 & 101 & 6 & -0.029 & -0.056 \\
\hline 101 & 11 & 7 & -0.052 & -0.034 \\
\hline $1 \mathrm{~b} 1$ & 101 & 8 & 0.048 & 0.079 \\
\hline 151 & 151 & 9 & 0.050 & 0.062 \\
\hline 101 & 1 [ 1 & 10 & -0.062 & -0.099 \\
\hline
\end{tabular}

Figure 4. Quarterly poliomyelitis series: the sample autocorrelations and partial autocorrelations

The data are thus firstly fitted by the ZT Poisson INARCH(2) and Poisson INARCH(2) models. Regarding the general good performance of the $\operatorname{GARCH}(1,1)$ modeling, we get also the ZT Poisson INGARCH(1,1) and Poisson INGARCH(1,1) fittings. Conditional maximum likelihood parameter estimates, their standard errors and the three criteria previously used are summarized in Table 5 . Despite the better values for the three criteria in the $\operatorname{INGARCH}(1,1)$ model we note the non significance of $\beta_{1}$ estimate. So the parsimonious principle lead us to consider an INARCH(1) modeling (lines 6 and 7) and we verify that the ZT Poisson INARCH(1) model presents the best results.

To analyze in practice the alternative of replacing a zero truncated model $Z$ by the corresponding shifted model $Z-1$, the fitting of a Poisson INARCH(1) model to the shifted series is considered (last line of Table 5). We note that, according to the criteria, this model gives the worst fitting. A ZT geometric INARCH(1) model was also fitted to this data. The results obtained (Table 6 in Appendix D) also show clearly that this is not the right model.

As an overall appreciation of the results of the Table 5, we point out that taking into consideration the Akaike and Schwarz criteria and the values of the log-likelihood function, the better performance of the ZT models is evident.

Finally, we observe that the improvement achieved with this ZT models related to Poisson distribution in the quarterly poliomyelitis data fitting is naturally due to the nature of this data that describe a rare phenomenon which occurs effectively with some 
Table 5. Conditional maximum likelihood estimates of the parameters, with the corresponding standard errors and probabilities, the log-likelihood function and Akaike and Schwarz criteria for the quarterly poliomyelitis cases.

\begin{tabular}{|c|c|c|c|c|c|c|c|}
\hline Model & \multicolumn{4}{|c|}{ Estimates } & \multirow{2}{*}{$\begin{array}{c}\log \mathrm{L} \\
89.5949\end{array}$} & \multirow{2}{*}{$\begin{array}{l}\begin{array}{l}\text { Akaike } \\
\text { criterion }\end{array} \\
-3.2072\end{array}$} & \multirow{2}{*}{$\begin{array}{l}\begin{array}{l}\text { Schwarz } \\
\text { criterion }\end{array} \\
-3.0967\end{array}$} \\
\hline ZT Poisson INARCH(2) & $\begin{array}{l}\widehat{\alpha}_{0} \\
\widehat{\alpha}_{1} \\
\widehat{\alpha}_{2}\end{array}$ & $\begin{array}{l}\text { Coeff. } \\
1.7785 \\
0.2822 \\
0.2245 \\
\end{array}$ & $\begin{array}{l}\text { Std. Error } \\
0.3232 \\
0.0520 \\
0.0545 \\
\end{array}$ & $\begin{array}{l}\text { Prob. } \\
0.0000 \\
0.0000 \\
0.0000 \\
\end{array}$ & & & \\
\hline Poisson INARCH(2) & $\begin{array}{l}\widehat{\alpha}_{0} \\
\widehat{\alpha}_{1} \\
\widehat{\alpha}_{2}\end{array}$ & $\begin{array}{l}\text { Coeff. } \\
2.0432 \\
0.2646 \\
0.2071\end{array}$ & $\begin{array}{l}\text { Std. Error } \\
0.3449 \\
0.0529 \\
0.0567\end{array}$ & $\begin{array}{l}\text { Prob. } \\
0.0000 \\
0.0000 \\
0.0003\end{array}$ & 87.5311 & -3.1308 & -3.0882 \\
\hline ZT Poisson INGARCH $(1,1)$ & $\begin{array}{l}\widehat{\alpha}_{0} \\
\widehat{\alpha}_{1} \\
\widehat{\beta}_{1}\end{array}$ & $\begin{array}{l}\text { Coeff. } \\
3.0840 \\
0.2558 \\
-0.0223 \\
\end{array}$ & $\begin{array}{l}\text { Std. Error } \\
0.4070 \\
0.0534 \\
0.1072 \\
\end{array}$ & $\begin{array}{l}\text { Prob. } \\
0.0000 \\
0.0000 \\
0.8351 \\
\end{array}$ & 97.6241 & -3.4409 & -3.3314 \\
\hline Poisson INGARCH(1,1) & $\begin{array}{l}\widehat{\alpha}_{0} \\
\widehat{\alpha}_{1} \\
\widehat{\beta}_{1}\end{array}$ & $\begin{array}{l}\text { Coeff. } \\
3.3869 \\
0.2387 \\
-0.0581\end{array}$ & $\begin{array}{l}\text { Std. Error } \\
0.4454 \\
0.0540 \\
0.1150\end{array}$ & $\begin{array}{l}\text { Prob. } \\
0.0000 \\
0.0000 \\
0.6135\end{array}$ & 96.3856 & -3.3958 & -3.2863 \\
\hline ZT Poisson INARCH(1) & $\begin{array}{l}\widehat{\alpha}_{0} \\
\widehat{\alpha}_{1}\end{array}$ & $\begin{array}{l}\text { Coeff. } \\
2.9808 \\
0.2591\end{array}$ & $\begin{array}{l}\text { Std. Error } \\
0.2118 \\
0.0501\end{array}$ & $\begin{array}{l}\text { Prob. } \\
0.0000 \\
0.0000\end{array}$ & 97.6201 & -3.4771 & -3.4041 \\
\hline Poisson INARCH(1) & $\begin{array}{l}\widehat{\alpha}_{0} \\
\widehat{\alpha}_{1}\end{array}$ & $\begin{array}{l}\text { Coeff. } \\
3.1156 \\
0.2472\end{array}$ & $\begin{array}{l}\text { Std. Error } \\
0.2180 \\
0.0507\end{array}$ & $\begin{array}{l}\text { Prob. } \\
0.0000 \\
0.0000\end{array}$ & 96.3591 & -3.4312 & -3.3582 \\
\hline Z-1: Poisson INARCH(1) & $\begin{array}{l}\widehat{\alpha}_{0} \\
\widehat{\alpha}_{1}\end{array}$ & $\begin{array}{l}\text { Coeff. } \\
2.3505 \\
0.2514\end{array}$ & $\begin{array}{l}\text { Std. Error } \\
0.1329 \\
0.0407\end{array}$ & $\begin{array}{l}\text { Prob. } \\
0.0000 \\
0.0000\end{array}$ & 27.6519 & -0.9328 & -0.8598 \\
\hline
\end{tabular}

regularity.

\section{Conclusion}

Compound Poisson INGARCH processes are a wide family of integer-valued models, recently introduced, that are able to describe simultaneously characteristics of count data like different kinds of conditional heteroscedasticity or overdispersion. But, these kind of processes all assume that the count data in analysis have zero counts. Many times the count systems to be modeled structurally exclude zeros. So, in order to model such data properly, the underlying probability distribution should preclude null outcomes. The potential of compound Poisson probability distributions to describe huge different characteristics of count data justify the introduction of the new class of zero truncated models inspired in the CP-INGARCH ones but amended to exclude zeros. We point out that the main subfamily of models here studied, namely the class of ZTCP-INGARCH processes with $\varphi_{t}$ deterministic and independent of $t$, may accommodate a significant number of models useful in applications. As relevant examples we should refer the ZT Poisson and the ZT Neyman Type A ones, corresponding respectively to $\varphi_{t}(u)=\exp (i u)$ and $\varphi_{t}(u)=\exp (\phi(\exp (i u)-1))$.

In conclusion, the probabilistic and statistical study developed is enough consistent for using these models in applications related to rare phenomena but with effective occurrences. We hope to have illustrated these facts with the simulation and real data studies presented. 


\section{Acknowledgements}

The authors thank the Referees and the Associate Editor for very useful comments on an earlier draft of this paper.

This work was partially supported by the Centre for Mathematics of the University of Coimbra - UID/MAT/00324/2013, funded by the Portuguese Government through FCT/MEC and co-funded by the European Regional Development Fund through the Partnership Agreement PT2020.

\section{Appendix A. Conditional ML estimation of ZT Poisson INGARCH models}

In order to estimate the asymptotic covariance matrix of the conditional maximum likelihood estimator, we begin by deriving the first and second derivatives of

$$
l_{t}(\theta)=Z_{t} \log \left(\lambda_{t}\right)-\lambda_{t}-\log \left(Z_{t} !\right)-\log \left(1-\exp \left(-\lambda_{t}\right)\right)
$$

in order to $\theta_{i}, i=0, \ldots, p+q$. The first derivatives of $l_{t}$ are

$$
\begin{aligned}
\frac{\partial l_{t}}{\partial \theta_{i}} & =Z_{t} \frac{\partial \lambda_{t}}{\partial \theta_{i}} \frac{1}{\lambda_{t}}-\frac{\partial \lambda_{t}}{\partial \theta_{i}}-\frac{-\exp \left(-\lambda_{t}\right)\left(-\frac{\partial \lambda_{t}}{\partial \theta_{i}}\right)}{1-\exp \left(-\lambda_{t}\right)} \\
& =\frac{\partial \lambda_{t}}{\partial \theta_{i}}\left(\frac{Z_{t}}{\lambda_{t}}-\frac{1}{1-\exp \left(-\lambda_{t}\right)}\right)
\end{aligned}
$$

and the second derivatives are

$$
\begin{aligned}
\frac{\partial^{2} l_{t}}{\partial \theta_{i} \partial \theta_{j}} & =\frac{\partial^{2} \lambda_{t}}{\partial \theta_{i} \partial \theta_{j}}\left(\frac{Z_{t}}{\lambda_{t}}-\frac{1}{1-\exp \left(-\lambda_{t}\right)}\right)+\frac{\partial \lambda_{t}}{\partial \theta_{i}}\left[-\frac{Z_{t}}{\lambda_{t}^{2}} \frac{\partial \lambda_{t}}{\partial \theta_{j}}+\left(1-\exp \left(-\lambda_{t}\right)\right)^{-2} \exp \left(-\lambda_{t}\right) \frac{\partial \lambda_{t}}{\partial \theta_{j}}\right] \\
& =\frac{\partial^{2} \lambda_{t}}{\partial \theta_{i} \partial \theta_{j}}\left(\frac{Z_{t}}{\lambda_{t}}-\frac{1}{1-\exp \left(-\lambda_{t}\right)}\right)+\left[-\frac{Z_{t}}{\lambda_{t}^{2}}+\frac{\exp \left(-\lambda_{t}\right)}{\left(1-\exp \left(-\lambda_{t}\right)\right)^{2}}\right] \frac{\partial \lambda_{t}}{\partial \theta_{i}} \frac{\partial \lambda_{t}}{\partial \theta_{j}}
\end{aligned}
$$

for $0 \leq i, j \leq p+q$. Moreover,

$$
\begin{aligned}
\frac{\partial \lambda_{t}}{\partial \alpha_{0}} & =1+\sum_{k=1}^{q} \beta_{k} \frac{\partial \lambda_{t-k}}{\partial \alpha_{0}} ; \\
\frac{\partial \lambda_{t}}{\partial \alpha_{i}} & =Z_{t-i}+\sum_{k=1}^{q} \beta_{k} \frac{\partial \lambda_{t-k}}{\partial \alpha_{i}}, i=1, \ldots, p ; \\
\frac{\partial \lambda_{t}}{\partial \beta_{j}} & =\lambda_{t-j}+\sum_{k=1}^{q} \beta_{k} \frac{\partial \lambda_{t-k}}{\partial \beta_{j}}, j=1, \ldots, q .
\end{aligned}
$$

Let us consider

$$
W_{t}^{T}=\left(1, Z_{t-1}, \ldots, Z_{t-p}, \lambda_{t-1}, \ldots, \lambda_{t-q}\right)
$$

and let $\nabla f$ denote the gradient of any function $f$. We have

$$
\nabla \lambda_{t}=W_{t}+\sum_{k=1}^{q} \beta_{k} \nabla \lambda_{t-k}
$$


The equation (2) may now be written as

$$
\nabla l_{t}=\left(\frac{Z_{t}}{\lambda_{t}}-\frac{1}{1-\exp \left(-\lambda_{t}\right)}\right) \nabla \lambda_{t}
$$

and the equation (3) becomes

$$
\mathbf{H}_{t}=\left(\frac{Z_{t}}{\lambda_{t}}-\frac{1}{1-\exp \left(-\lambda_{t}\right)}\right) \nabla\left(\nabla^{T} \lambda_{t}\right)-\left[\frac{Z_{t}}{\lambda_{t}^{2}}-\frac{\exp \left(-\lambda_{t}\right)}{\left(1-\exp \left(-\lambda_{t}\right)\right)^{2}}\right] \nabla \lambda_{t} \nabla^{T} \lambda_{t} .
$$

Taking expectations in both sides of the equation (3) we obtain

$$
\begin{aligned}
& E\left(\frac{\partial^{2} l_{t}}{\partial \theta_{i} \partial \theta_{j}} \underline{Z}_{t-1}\right)= \\
& \quad=E\left[\frac{\partial^{2} \lambda_{t}}{\partial \theta_{i} \partial \theta_{j}}\left(\frac{Z_{t}}{\lambda_{t}}-\frac{1}{1-\exp \left(-\lambda_{t}\right)}\right)-\left(\frac{Z_{t}}{\lambda_{t}^{2}}-\frac{\exp \left(-\lambda_{t}\right)}{\left(1-\exp \left(-\lambda_{t}\right)\right)^{2}}\right) \frac{\partial \lambda_{t}}{\partial \theta_{i}} \frac{\partial \lambda_{t}}{\partial \theta_{j}} \mid \underline{Z}_{t-1}\right] \\
& \quad=\frac{\partial^{2} \lambda_{t}}{\partial \theta_{i} \partial \theta_{j}} E\left(\frac{Z_{t}}{\lambda_{t}}-\frac{1}{1-\exp \left(-\lambda_{t}\right)} \mid \underline{Z}_{t-1}\right)-E\left(\frac{Z_{t}}{\lambda_{t}^{2}}-\frac{\exp \left(-\lambda_{t}\right)}{\left(1-\exp \left(-\lambda_{t}\right)\right)^{2}} \mid \underline{Z}_{t-1}\right) \frac{\partial \lambda_{t}}{\partial \theta_{i}} \frac{\partial \lambda_{t}}{\partial \theta_{j}} .
\end{aligned}
$$

But from $E\left(Z_{t} \mid \underline{Z}_{t-1}\right)=\frac{\lambda_{t}}{1-\exp \left(-\lambda_{t}\right)}$ we deduce

$$
E\left(\frac{Z_{t}}{\lambda_{t}} \mid \underline{Z}_{t-1}\right)=\frac{1}{1-\exp \left(-\lambda_{t}\right)}
$$

and

$$
E\left(\frac{Z_{t}}{\lambda_{t}^{2}} \mid \underline{Z}_{t-1}\right)=\frac{1}{\lambda_{t}\left(1-\exp \left(-\lambda_{t}\right)\right)}
$$

So

$$
E\left(\frac{\partial^{2} l_{t}}{\partial \theta_{i} \partial \theta_{j}} \mid \underline{Z}_{t-1}\right)=-\left(\frac{1}{\lambda_{t}\left(1-\exp \left(-\lambda_{t}\right)\right)}-\frac{\exp \left(-\lambda_{t}\right)}{\left(1-\exp \left(-\lambda_{t}\right)\right)^{2}}\right) \frac{\partial \lambda_{t}}{\partial \theta_{i}} \frac{\partial \lambda_{t}}{\partial \theta_{j}} .
$$

Consequently

$$
-E\left(\frac{\partial^{2} l_{t}}{\partial \theta_{i} \partial \theta_{j}}\right)=E\left[\left(\frac{1}{\lambda_{t}\left(1-\exp \left(-\lambda_{t}\right)\right)}-\frac{\exp \left(-\lambda_{t}\right)}{\left(1-\exp \left(-\lambda_{t}\right)\right)^{2}}\right) \frac{\partial \lambda_{t}}{\partial \theta_{i}} \frac{\partial \lambda_{t}}{\partial \theta_{j}}\right] .
$$

In an analogous way, from (2) we get

$$
E\left(\frac{\partial l_{t}}{\partial \theta_{i}} \frac{\partial l_{t}}{\partial \theta_{j}} \mid \underline{Z}_{t-1}\right)=E\left(\frac{\partial \lambda_{t}}{\partial \theta_{i}} \frac{\partial \lambda_{t}}{\partial \theta_{j}}\left(\frac{Z_{t}}{\lambda_{t}}-\frac{1}{1-\exp \left(-\lambda_{t}\right)}\right)^{2} \mid \underline{Z}_{t-1}\right) .
$$

Taking into account that $E\left(Z_{t}^{2} \mid \underline{Z}_{t-1}\right)=\frac{\lambda_{t}^{2}+\lambda_{t}}{1-\exp \left(-\lambda_{t}\right)}$ we deduce

$$
\begin{aligned}
E\left(\frac{\partial l_{t}}{\partial \theta_{i}} \frac{\partial l_{t}}{\partial \theta_{j}} \mid \underline{Z}_{t-1}\right) & =\frac{\partial \lambda_{t}}{\partial \theta_{i}} \frac{\partial \lambda_{t}}{\partial \theta_{j}} E\left(\left(\frac{Z_{t}}{\lambda_{t}}\right)^{2}-\frac{2 Z_{t}}{\lambda_{t}\left(1-\exp \left(-\lambda_{t}\right)\right)}+\left(\frac{1}{1-\exp \left(-\lambda_{t}\right)}\right)^{2} \mid \underline{Z}_{t-1}\right) \\
& =\frac{\partial \lambda_{t}}{\partial \theta_{i}} \frac{\partial \lambda_{t}}{\partial \theta_{j}}\left[\frac{1+\frac{1}{\lambda_{t}}}{1-\exp \left(-\lambda_{t}\right)}-2\left(\frac{1}{1-\exp \left(-\lambda_{t}\right)}\right)^{2}+\left(\frac{1}{1-\exp \left(-\lambda_{t}\right)}\right)^{2}\right] \\
& =\frac{\partial \lambda_{t}}{\partial \theta_{i}} \frac{\partial \lambda_{t}}{\partial \theta_{j}}\left[\frac{\lambda_{t}+1}{\lambda_{t}\left(1-\exp \left(-\lambda_{t}\right)\right)}-\left(\frac{1}{1-\exp \left(-\lambda_{t}\right)}\right)^{2}\right] .
\end{aligned}
$$


Consequently

$$
\begin{aligned}
E\left(\frac{\partial l_{t}}{\partial \theta_{i}} \frac{\partial l_{t}}{\partial \theta_{j}}\right) & =E\left[\frac{\partial \lambda_{t}}{\partial \theta_{i}} \frac{\partial \lambda_{t}}{\partial \theta_{j}}\left(\frac{\lambda_{t}+1}{\lambda_{t}\left(1-\exp \left(-\lambda_{t}\right)\right)}-\frac{1}{\left(1-\exp \left(-\lambda_{t}\right)\right)^{2}}\right)\right] \\
& =E\left[\frac{\partial \lambda_{t}}{\partial \theta_{i}} \frac{\partial \lambda_{t}}{\partial \theta_{j}}\left(\frac{1}{1-\exp \left(-\lambda_{t}\right)}+\frac{1}{\lambda_{t}\left(1-\exp \left(-\lambda_{t}\right)\right)}-\frac{1}{\left(1-\exp \left(-\lambda_{t}\right)\right)^{2}}\right)\right] \\
& =E\left[\frac{\partial \lambda_{t}}{\partial \theta_{i}} \frac{\partial \lambda_{t}}{\partial \theta_{j}}\left(\frac{-\exp \left(-\lambda_{t}\right)}{\left(1-\exp \left(-\lambda_{t}\right)\right)^{2}}+\frac{1}{\lambda_{t}\left(1-\exp \left(-\lambda_{t}\right)\right)}\right)\right]
\end{aligned}
$$

We deduce that this ZT model satisfy the information matrix equality

$$
-E\left(\frac{\partial^{2} l_{t}}{\partial \theta_{i} \partial \theta_{j}}\right)=E\left(\frac{\partial l_{t}}{\partial \theta_{i}} \frac{\partial l_{t}}{\partial \theta_{j}}\right)
$$

\section{Appendix B. Conditional ML estimation of a ZT geometric INGARCH model}

Let us consider the estimation of the asymptotic covariance matrix of the conditional maximum likelihood estimator, $\widehat{\theta}$, of a ZT geometric INGARCH model. The first derivatives of $l_{t}(\theta)=-\log \lambda_{t}+Z_{t}\left[\log \lambda_{t}-\log \left(1+\lambda_{t}\right)\right]$ in order to $\theta_{i}, i=0, \ldots, p+q$, are

$$
\begin{aligned}
\frac{\partial l_{t}}{\partial \theta_{i}} & =-\frac{1}{\lambda_{t}} \frac{\partial \lambda_{t}}{\partial \theta_{i}}+Z_{t}\left[\frac{1}{\lambda_{t}} \frac{\partial \lambda_{t}}{\partial \theta_{i}}-\frac{1}{1+\lambda_{t}} \frac{\partial \lambda_{t}}{\partial \theta_{i}}\right] \\
& =\frac{\partial \lambda_{t}}{\partial \theta_{i}}\left[-\frac{1}{\lambda_{t}}+Z_{t} \frac{1}{\lambda_{t}\left(1+\lambda_{t}\right)}\right]
\end{aligned}
$$

and the second derivatives

$$
\begin{aligned}
\frac{\partial^{2} l_{t}}{\partial \theta_{i} \partial \theta_{j}} & =\frac{\partial^{2} \lambda_{t}}{\partial \theta_{i} \partial \theta_{j}}\left[-\frac{1}{\lambda_{t}}+Z_{t} \frac{1}{\lambda_{t}\left(1+\lambda_{t}\right)}\right]+\frac{\partial \lambda_{t}}{\partial \theta_{i}}\left[\frac{1}{\lambda_{t}^{2}} \frac{\partial \lambda_{t}}{\partial \theta_{j}}+Z_{t} \frac{-\frac{\partial \lambda_{t}}{\partial \theta_{j}}\left(1+\lambda_{t}\right)-\lambda_{t} \frac{\partial \lambda_{t}}{\partial \theta_{j}}}{\lambda_{t}^{2}\left(1+\lambda_{t}\right)^{2}}\right] \\
& =\frac{\partial^{2} \lambda_{t}}{\partial \theta_{i} \partial \theta_{j}}\left[-\frac{1}{\lambda_{t}}+Z_{t} \frac{1}{\lambda_{t}\left(1+\lambda_{t}\right)}\right]+\frac{\partial \lambda_{t}}{\partial \theta_{i}} \frac{\partial \lambda_{t}}{\partial \theta_{j}}\left[\frac{1}{\lambda_{t}^{2}}-Z_{t} \frac{1+2 \lambda_{t}}{\lambda_{t}^{2}\left(1+\lambda_{t}\right)^{2}}\right]
\end{aligned}
$$

for $0 \leq i, j \leq p+q$. Moreover, $\frac{\partial \lambda_{t}}{\partial \alpha_{0}}, \frac{\partial \lambda_{t}}{\partial \alpha_{i}}, i=1, \ldots, p$, and $\frac{\partial \lambda_{t}}{\partial \beta_{j}}, j=1, \ldots, q$, satisfy the relations presented in Appendix A. Setting

$$
W_{t}^{T}=\left(1, Z_{t-1}, \ldots, Z_{t-p}, \lambda_{t-1}, \ldots, \lambda_{t-q}\right)
$$

and denoting $\nabla f$ the gradient of any function $f$, we have

$$
\nabla \lambda_{t}=W_{t}+\sum_{k=1}^{q} \beta_{k} \nabla \lambda_{t-k}
$$

The equations (2) and (3) may now be written as

$$
\begin{gathered}
\nabla l_{t}=\left(-\frac{1}{\lambda_{t}}+Z_{t} \frac{1}{\lambda_{t}\left(1+\lambda_{t}\right)}\right) \nabla \lambda_{t} \\
\mathbf{H}_{t}=\left(-\frac{1}{\lambda_{t}}+Z_{t} \frac{1}{\lambda_{t}\left(1+\lambda_{t}\right)}\right) \nabla\left(\nabla^{T} \lambda_{t}\right)-\left[\frac{1}{\lambda_{t}^{2}}-Z_{t} \frac{1+2 \lambda_{t}}{\lambda_{t}^{2}\left(1+\lambda_{t}\right)^{2}}\right] \nabla \lambda_{t} \nabla^{T} \lambda_{t} .
\end{gathered}
$$


Like in the previous Appendix we obtain

$$
\begin{gathered}
E\left(\frac{\partial^{2} l_{t}}{\partial \theta_{i} \partial \theta_{j}} \mid \underline{Z}_{t-1}\right)=E\left[\frac{\partial^{2} \lambda_{t}}{\partial \theta_{i} \partial \theta_{j}}\left(-\frac{1}{\lambda_{t}}+Z_{t} \frac{1}{\lambda_{t}\left(1+\lambda_{t}\right)}\right)-\left(\frac{1}{\lambda_{t}^{2}}-Z_{t} \frac{1+2 \lambda_{t}}{\lambda_{t}^{2}\left(1+\lambda_{t}\right)^{2}}\right) \frac{\partial \lambda_{t}}{\partial \theta_{i}} \frac{\partial \lambda_{t}}{\partial \theta_{j}} \mid \underline{Z}_{t-1}\right] \\
=\frac{\partial^{2} \lambda_{t}}{\partial \theta_{i} \partial \theta_{j}} E\left(-\frac{1}{\lambda_{t}}+Z_{t} \frac{1}{\lambda_{t}\left(1+\lambda_{t}\right)} \mid \underline{Z}_{t-1}\right)-E\left(\frac{1}{\lambda_{t}^{2}}-Z_{t} \frac{1+2 \lambda_{t}}{\lambda_{t}^{2}\left(1+\lambda_{t}\right)^{2}} \mid \underline{Z}_{t-1}\right) \frac{\partial \lambda_{t}}{\partial \theta_{i}} \frac{\partial \lambda_{t}}{\partial \theta_{j}}, \\
E\left(\frac{\partial^{2} l_{t}}{\partial \theta_{i} \partial \theta_{j}} \mid \underline{Z}_{t-1}\right)=-\left(\frac{1}{\lambda_{t}^{2}}-\frac{1+2 \lambda_{t}}{\lambda_{t}^{2}\left(1+\lambda_{t}\right)}\right) \frac{\partial \lambda_{t}}{\partial \theta_{i}} \frac{\partial \lambda_{t}}{\partial \theta_{j}}=\frac{1}{\lambda_{t}\left(1+\lambda_{t}\right)} \frac{\partial \lambda_{t}}{\partial \theta_{i}} \frac{\partial \lambda_{t}}{\partial \theta_{j}},
\end{gathered}
$$

and so

$$
-E\left(\frac{\partial^{2} l_{t}}{\partial \theta_{i} \partial \theta_{j}}\right)=E\left[\frac{1}{\lambda_{t}\left(1+\lambda_{t}\right)} \frac{\partial \lambda_{t}}{\partial \theta_{i}} \frac{\partial \lambda_{t}}{\partial \theta_{j}}\right]
$$

We also get

$$
E\left(\frac{\partial l_{t}}{\partial \theta_{i}} \frac{\partial l_{t}}{\partial \theta_{j}} \mid \underline{Z}_{t-1}\right)=E\left(\frac{\partial \lambda_{t}}{\partial \theta_{i}} \frac{\partial \lambda_{t}}{\partial \theta_{j}}\left(-\frac{1}{\lambda_{t}}+Z_{t} \frac{1}{\lambda_{t}\left(1+\lambda_{t}\right)}\right)^{2} \mid \underline{Z}_{t-1}\right),
$$

and taking into account that $E\left(Z_{t}^{2} \mid \underline{Z}_{t-1}\right)=\left(2 \lambda_{t}+1\right)\left(1+\lambda_{t}\right)$ we deduce

$$
\begin{aligned}
E\left(\frac{\partial l_{t}}{\partial \theta_{i}} \frac{\partial l_{t}}{\partial \theta_{j}} \mid \underline{Z}_{t-1}\right) & =\frac{\partial \lambda_{t}}{\partial \theta_{i}} \frac{\partial \lambda_{t}}{\partial \theta_{j}} E\left(\frac{1}{\lambda_{t}^{2}}-\frac{2 Z_{t}}{\lambda_{t}^{2}\left(1+\lambda_{t}\right)}+Z_{t}^{2} \frac{1}{\lambda_{t}^{2}\left(1+\lambda_{t}\right)^{2}} \underline{Z}_{t-1}\right) \\
& =\frac{\partial \lambda_{t}}{\partial \theta_{i}} \frac{\partial \lambda_{t}}{\partial \theta_{j}}\left[\frac{1}{\lambda_{t}\left(1+\lambda_{t}\right)}\right] .
\end{aligned}
$$

The usual information matrix equality

$$
-E\left(\frac{\partial^{2} l_{t}}{\partial \theta_{i} \partial \theta_{j}}\right)=E\left(\frac{\partial l_{t}}{\partial \theta_{i}} \frac{\partial l_{t}}{\partial \theta_{j}}\right)
$$

also follows in the conditional maximum likelihood estimation of a ZT geometric INGARCH model.

\section{Appendix C. Algorithm of generating the data in Figures 1 and 2}

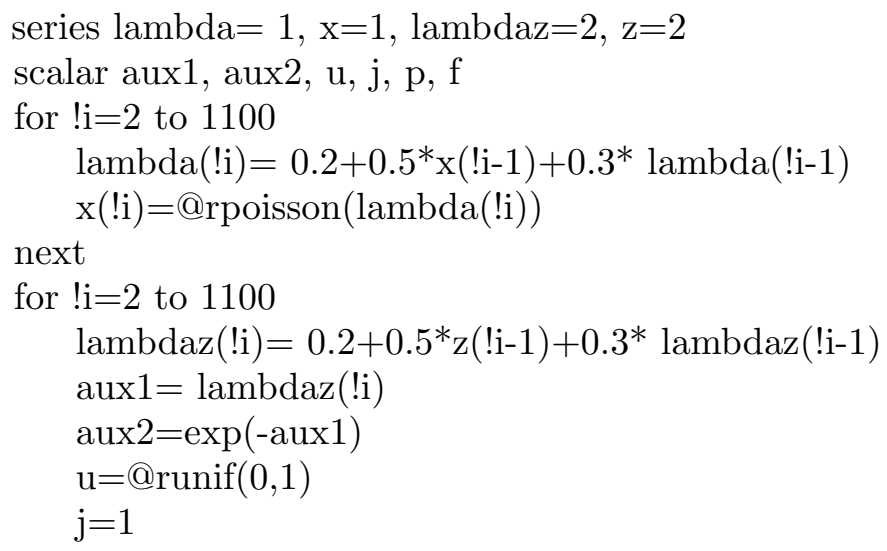




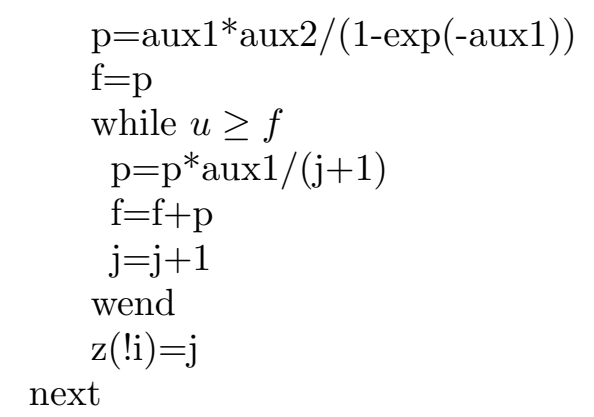

\section{Appendix D. Quarterly poliomyelitis data and the ZT geometric INARCH(1) model}

Table 6. Conditional maximum likelihood estimates of the parameters of the ZT geometric INARCH(1) model for the quarterly poliomyelitis data, with the corresponding standard errors and probabilities, the log-likelihood function and Akaike and Schwarz criteria.

\begin{tabular}{|c|c|c|c|c|c|c|c|}
\hline Model & \multicolumn{4}{|c|}{ Estimates } & Log L & Akaike & Schwarz \\
\hline ZT G INARCH(1) & $\begin{array}{l}\widehat{\alpha}_{0} \\
\widehat{\alpha}_{1}\end{array}$ & $\begin{array}{l}\text { Coeff. } \\
2.0456 \\
0.2268\end{array}$ & $\begin{array}{l}\text { Std. Error } \\
0.6172 \\
0.2156\end{array}$ & $\begin{array}{l}\text { Prob. } \\
0.0009 \\
0.2137\end{array}$ & -123.7018 & 4.5710 & 4.6440 \\
\hline
\end{tabular}

\section{References}

[1] Ahmad, A., Francq, Ch. (2016). Poisson QMLE of count time series models. Journal of Time Series Analysis, 37, 291-314.

[2] Bakouch, H.S., Ristic, M.M. (2010). Zero truncated Poisson integer-valued AR(1) model. Metrika, 72, 265-280.

[3] Francq, Ch. and Zakoian, J.M. (2010). GARCH models, Structure, Statistical inference and Financial applications. John Wiley and Sons.

[4] Gonçalves, E., Mendes-Lopes, N., and Silva, F. (2015). Infinitely divisible distributions in integer-valued GARCH models. Journal of Time Series Analysis, 36, 503-527.

[5] Gonçalves, E., Mendes-Lopes, N., and Silva, F. (2016). Zero-inflated compound Poisson distributions in integer-valued GARCH models. Statistics, 50, 558-578.

[6] Gouriéroux, Ch., Monfort, A., Trognon, A. (1984). Pseudo maximum likelihood methods: theory. Econometrica, 52, 681-700.

[7] Hilbe, J. M. (2014). Modeling Count Data. Cambridge University Press.

[8] Lee, S., Lee, Y., and Chen, C. (2015) Parameter change test for zero-inflated generalized Poisson autoregressive models. Statistics, page DOI:10.1080/02331888.2015.1083020.

[9] Zhu, F. (2011). A negative binomial integer-valued GARCH model. Journal of Time Series Analysis, 32, 54-67.

[10] Zhu, F. (2012). Zero-inflated Poisson and negative binomial integer-valued GARCH models. J. Stat. Plann. and Inf., 142, 826-839. 